\title{
Practical Challenges and Methodological Flexibility in Prior Elicitation
}

\author{
Angelika M. Stefan ${ }^{a}$, Nathan J. Evans ${ }^{a, b}$, Eric-Jan Wagenmakers $^{a}$ \\ ${ }^{a}$ Department of Psychology, University of Amsterdam, The Netherlands \\ ${ }^{b}$ School of Psychology, University of Queensland, Australia
}

This is an unedited manuscript accepted for publication in Psychological Methods.

The published version of the paper can be accessed under the following link:

https://doi.org/10.1037/met0000354.

Correspondence may be addressed to a.m.stefan@uva.nl (AMS).

Associated materials can be found at https://osf.io/tbz6n/.

Prior dissemination: Parts of this manuscript have been presented by AMS at the 2019 Meeting of the European Mathematical Psychology Group (EMPG). A preprint of this manuscript has been published on https://psyarxiv.com/d42xb/.

Funding information: AMS was supported by the NWO Research Talent Programme (406.18.556). NJE was supported by an Australian Research Council Discovery Early Career Researcher Award (DE200101130). EJW was supported by a European Research Council Advanced Grant (743086 UNIFY). 


\begin{abstract}
The Bayesian statistical framework requires the specification of prior distributions, which reflect pre-data knowledge about the relative plausibility of different parameter values. As prior distributions influence the results of Bayesian analyses, it is important to specify them with care. Prior elicitation has frequently been proposed as a principled method for deriving prior distributions based on expert knowledge. Although prior elicitation provides a theoretically satisfactory method of specifying prior distributions, there are several implicit decisions that researchers need to make at different stages of the elicitation process, each of them constituting important researcher degrees of freedom. Here, we discuss some of these decisions and group them into three categories: decisions about (1) the setup of the prior elicitation; (2) the core elicitation process; and (3) combination of elicited prior distributions from different experts. Importantly, different decision paths could result in greatly varying priors elicited from the same experts. Hence, researchers who wish to perform prior elicitation are advised to carefully consider each of the practical decisions before, during, and after the elicitation process. By explicitly outlining the consequences of these practical decisions, we hope to raise awareness for methodological flexibility in prior elicitation and provide researchers with a more structured approach to navigate the decision paths in prior elicitation. Making the decisions explicit also provides the foundation for further research that can identify evidence-based best practices that may eventually reduce the methodologically flexibility in prior elicitation.
\end{abstract}




\section{Translational Abstract}

Psychologists often use statistical models to evaluate data collected from psychological studies. Knowledge from field experts, such as psychotherapists, can be used to make these statistical models more diagnostic. The knowledge can be obtained using prior elicitation, an interview method that allows researchers to express the experts' knowledge in terms of a statistical distribution. Here, we show that researchers need to make many practical decisions in prior elicitation. Importantly, different decision paths could result in different prior elicitation results. Hence, we argue that researchers who wish to perform prior elicitation should carefully consider each of the practical decisions before, during, and after the elicitation process. By explicitly outlining potential consequences of the decisions, we hope to raise awareness of the inherent flexibility in prior elicitation procedures and provide researchers with a more structured approach to navigate the decision paths in prior elicitation. Making the decisions explicit also provides the foundation for further research that can identify evidence-based practices for prior elicitation.

\section{Keywords}

Prior distribution; prior elicitation; informative prior; methodological flexibility; Bayesian methods 
Prior distributions are an indispensable part of the Bayesian statistical framework. However, opinions differ as to whether priors should be seen as a necessary evil or an attractive feature of Bayesian methods. On the one hand, prior distributions have been fervently criticized for their perceived inherent subjectiveness (e.g., Efron 1986; but see Lindley 1986). Even some proponents of Bayesian methods have expressed concerns that poorly specified prior distributions may bring an "excellent model [...] under suspicion" (Bayarri \& Berger, 2000), and may undermine the validity of marginal likelihoods as a measure of evidence (Liu \& Aitkin, 2008; Kass \& Raftery, 1995). On the other hand, prior distributions have been proposed as a powerful tool to incorporate existing information into statistical models (Lindley, 2004; O'Hagan, 2019). By constraining model predictions to realistic scenarios (Gelman et al., 2017), carefully specified prior distributions can ensure that statistical models provide an accurate reflection of substantive theories (Lee \& Vanpaemel, 2017; Dienes, 2011; Vanpaemel, 2010). Consequently, models with carefully specified prior distributions that incorporate available substantive knowledge can yield more diagnostic and informative tests of theory (Gronau et al., in press).

Two essential insights can be distilled from the recurring debates on prior specification. Firstly, there is no way around prior specification in Bayesian statistics. Regardless of their personal stand on prior distributions, all researchers employing Bayesian methods must specify them. Secondly, prior distributions can considerably influence the results of Bayesian model comparisons and parameter estimation. This makes it necessary to justify the choice of prior distributions in the context of model specification.

One method to specify prior distributions is through prior elicitation from experts (e.g., Dias et al., 2018). Prior elicitation is a structured interview process designed to transform qualitative probability judgments of subject experts into quantitative probability distributions. Enriching statistical models with relevant expert knowledge is often practically desirable because it allows for realistic predictions and diagnostic tests in the face of sparse data (Stefan et al., 2019).

Given that data is often costly to obtain or subjected to limited availability, is not 
surprising that more and more statisticians consider enriching their statistical models with expert knowledge through prior elicitation from experts. For example, of the 59 articles published in the journal Bayesian Analysis in 2019 and 2020 until today (March 2020), 7 articles discuss in detail how results of prior elicitation could be used in Bayesian modeling and 9 additional articles at least mention prior elicitation from experts. Prior elicitation is gaining popularity in many different fields such as economics, medicine, or engineering (for an overview, see O'Hagan et al., 2006) and is already performed routinely in some areas, for example in pharmaceutical research (e.g., Thall \& Cook, 2004; Thall et al., 2017; Guo et al., 2019; Ursino et al., 2019). In psychology, there have been relatively few prior elicitation efforts (for exceptions see Gronau et al., in press; Bolsinova et al., 2017; Tessler \& Goodman, 2019; Sarma \& Kay, 2020), but given the increasing use of Bayesian methods (Andrews \& Baguley, 2013; Tendeiro \& Kiers, 2019) and the broad support for informed Bayesian methods by Bayesian experts (Aczel et al., in press), it seems plausible that prior elicitation may also become widely adopted in psychology in the future.

In this paper, we examine several practical decisions that researchers need to make in every prior elicitation procedure. We believe that highlighting these decisions is important as researchers are often unaware of these decisions and often make them without a sufficient evidential basis. Over the course of this paper, we demonstrate that many of these practical decisions can have a substantial impact on the results of the prior elicitation procedure, leading to considerable researcher degrees of freedom. We discuss several practical and theoretical considerations researchers can make when faced with these decisions, and give preliminary directions as to how researchers can manage the multiplicity of results stemming from methodological flexibility in the prior elicitation process. Our paper also motivates further research aimed at establishing evidence-based gold standards for prior elicitation.

After giving a short explanation of the role of the prior distribution in Bayesian inference, we will provide a brief introduction to prior elicitation in the context of subjective Bayesian analyses. In the remainder of the article, we will discuss decisions that need to be taken before, during, and after a prior elicitation procedure. We will begin by outlining the 
decisions involving the choice of experts and parameters for the elicitation. Then, we will discuss decisions about the core interview process, such as which prior elicitation technique should be used and how the elicitation results should be transformed into a probability distribution. Finally, we address the question of whether and how prior distributions elicited from many experts should be combined. Based on the discussed practical problems of prior elicitation, we derive several preliminary recommendations for prior elicitation and conclude that transparency and a broader empirical basis will be necessary to increase the practical usefulness and methodological rigor of prior elicitation in the future.

\section{Prior Distributions in Bayesian Inference}

Prior distributions are probability distributions that are placed on parameter values in Bayesian statistical models. The shape of the distribution represents the relative plausibility of parameter values prior to data collection. Therefore, prior distributions are typically specified before the data collection takes place. ${ }^{1}$ The knowledge incorporated in the prior distribution is updated based on observed data. Mathematically, this is described by Bayes' rule as an update from the prior distribution to the posterior distribution:

$$
\underbrace{p(\theta \mid \mathcal{D})}_{\text {Posterior }}=\underbrace{p(\theta)}_{\text {Prior }} \times \frac{\overbrace{p(\mathcal{D} \mid \theta)}^{\text {Likelihood }}}{\underbrace{p(\mathcal{D})}_{\text {Marginal Likelihood }}},
$$

where $p(\theta \mid \mathcal{D})$ is the posterior distribution of the parameter given the data $\mathcal{D}, p(\mathcal{D} \mid \theta)$ is the likelihood of the data given the parameter values $\theta, p(\theta)$ is the prior distribution of the parameter, and $p(\mathcal{D})$ is the marginal likelihood of the data. The primary focus in Bayesian parameter estimation concerns the posterior distribution which represents the updated knowledge about a parameter in a model after seeing the data. As shown in Equation 1, the shape of the prior distribution directly influences the shape of the posterior distribution.

\footnotetext{
${ }^{1}$ But see empirical Bayes approaches that define the prior based on data (e.g., Casella, 1985)
} 
Another key interest in Bayesian inference is to assess the relative support that the data provide for each of a series of rival models. This can be achieved by a Bayesian model comparison. In Bayesian model comparisons, the primary concern is the marginal likelihood of the data $p(\mathcal{D})$ under each model $\left(\mathcal{M}_{i}\right)$. The marginal likelihood can be found by integrating over the parameter space - that is, all parameter values that are logically possible under the respective model - and can be interpreted as a measure of the predictive performance of the model,

$$
p\left(\mathcal{D} \mid \mathcal{M}_{i}\right)=\int p\left(\mathcal{D} \mid \theta_{i}, \mathcal{M}_{i}\right) p\left(\theta_{i} \mid \mathcal{M}_{i}\right) \mathrm{d} \theta
$$

As can be seen from Equation 2, the prior distribution $p(\theta \mid \mathcal{M})$ plays an important role in the calculation of the marginal likelihood of a model, where changes in the prior distribution will result in changes of the marginal likelihood. The ratio of two marginal likelihoods is called a Bayes factor and provides a measure of relative evidence provided by the data for one model over the other model (Kass \& Raftery, 1995):

$$
\mathrm{BF}_{12}=\frac{p\left(\mathcal{D} \mid \mathcal{M}_{1}\right)}{p\left(\mathcal{D} \mid \mathcal{M}_{2}\right)}
$$

The Bayes factor is a quantity of interest in Bayesian hypothesis testing, where a model representing the null hypothesis is compared to a model representing the alternative hypothesis. $^{2}$ These models are typically nested, such that a parameter is fixed in the null model and a prior distribution is put on the parameter in the alternative model (Jeffreys, 1961; Sinharay \& Stern, 2002). By definition, the result of the hypothesis test is sensitive to the prior distribution for the parameter in the alternative model. Therefore, a careful choice of prior distributions is essential in Bayesian hypothesis testing (Tendeiro \& Kiers, 2019; Vanpaemel, 2010; van Ravenzwaaij \& Wagenmakers, 2019; Jeffreys, 1939). Depending on the model parametrization, priors can be placed on raw values, for example a difference

\footnotetext{
${ }^{2}$ Note that tools other than the Bayes factor also allow comparing models (Evans, 2019), for example Bayesian leave-one-out cross validation (Gronau \& Wagenmakers, 2019), however, in this article we will focus on Bayesian null hypothesis testing with Bayes factors.
} 
in means (Dienes \& Mclatchie, 2017), or on standardized parameters, for example a standardized effect size (Rouder et al., 2009; Gronau et al., in press).

\section{Prior Elicitation as a Basis for Subjective Bayesian Analyses}

Among Bayesian statisticians, there has been a lively debate about what level of prior information should be incorporated in the prior distribution (e.g., Goldstein, 2006; Fienberg, 2006; J. Berger, 2006; Jeffreys, 1963). On the one hand, "objective" Bayesians aim to find default solutions for common statistical problems without the assumption of existing prior knowledge. They specify prior distributions with regard to one or more desirable mathematical properties, for example maximum entropy (Jaynes, 1982), maximal data information (Zellner \& Min, 1993), invariance under reparametrization (Hartigan, 1964), or information consistency of the Bayes factor (Ly et al., 2016; for overviews see Kass \& Raftery, 1995, Bayarri et al., 2012, and Consonni et al., 2018). Objective prior distributions are often used as a default in software packages for Bayesian inference, e.g., in the BayesFactor $R$ package (Morey \& Rouder, 2018) and in JASP (JASP Team, 2020).

On the other hand, "subjective" Bayesians claim that prior distributions should be informed by scientific theory (Vanpaemel, 2010; Lee \& Vanpaemel, 2017) and prior knowledge about parameters (Goldstein, 2006). ${ }^{3}$ The underlying idea is that the prior distribution is an integral part of a Bayesian statistical model and that quantitative predictions of the statistical model should be consistent with the expectations of field experts (Gelman et al., 2017). Following this logic, it is impossible to find a one-size-fits-all default prior distribution (Fienberg, 2006). Instead, custom "informed" prior distributions need to be defined for specific application problems. The focus of our article - prior elicitation - is a general approach for creating these informed prior distributions based on expert knowledge. However, we wish to note that our focus on prior elicitation is not an endorsement of the subjective Bayesian approach over the objective Bayesian approach, and readers interested in philosophical debates between proponents of these approaches should, for example, read

\footnotetext{
${ }^{3}$ Note that the terms "objective" and "subjective" Bayesian inference do not imply a normative relationship but are used as descriptive terms for different modeling frameworks.
} 

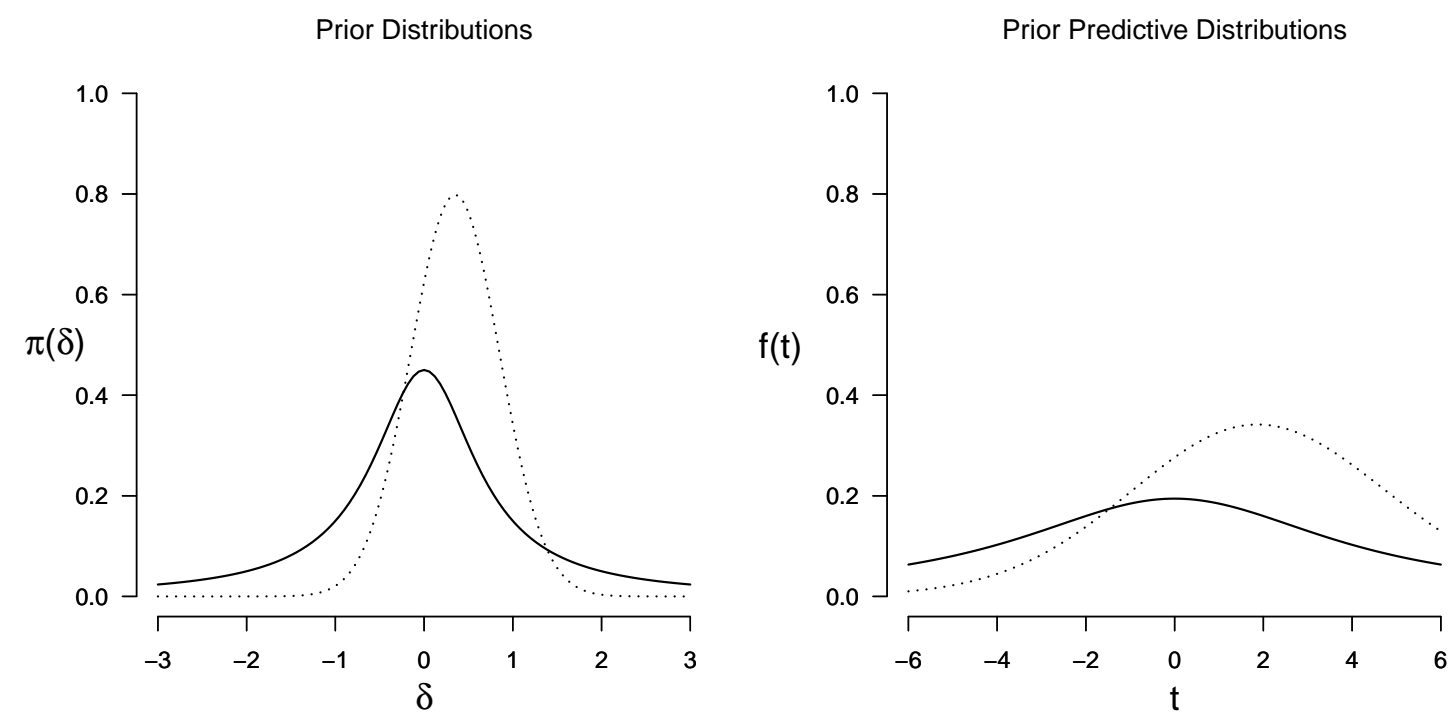

Figure 1. Two prior distributions on Cohen's $\delta$ in a Bayesian one-sample $t$-test and the respective prior predictive distributions for a sample size of $N=30$. Solid lines refer to the default prior (Cauchy distribution with location parameter $\mu=0$, scale parameter $\mathrm{r}=$ $\sqrt{2} / 2$; see Rouder et al., 2009), dotted lines refer to an informed prior (normal distribution with mean $\mu=0.35$ and variance $\sigma^{2}=0.25$; see Gronau et al., in press).

the informative exchange between Goldstein (2006), Fienberg (2006), and J. Berger (2006).

Figure 1 shows an example for the different quantitative predictions of a default and an informed prior distribution in practice. Specifically, Figure 1 displays two probability distributions that can be used as a prior distribution on the effect size Cohen's $\delta$ in the alternative hypothesis model of a Bayesian one-sample $t$-test. The Cauchy prior is an oftenused default prior that leads the test to fulfill the desiderata of predictive matching and information consistency (Rouder et al., 2009; Ly et al., 2016). The normal prior represents one option for a moderately informed prior distribution (Gronau et al., in press). The two prior distributions are shown in the left panel of Figure 1. The right panel of Figure 1 shows the likelihood of observing certain $t$-values under the default prior model and under the informed prior model, that is, the prior predictive distributions for both models. As can be seen, the Cauchy prior makes vague predictions, assigns the highest likelihood to $t=0$, and a relatively high likelihood to extreme values. This might not be aligned with 
researchers' predictions who often expect small, but non-zero, effects (Richard et al., 2003). Thus, an informed prior such as the one shown in Figure 1 may better represent their current state of knowledge and make more realistic predictions.

In a Bayesian hypothesis testing setting, the more constrained predictions of informed prior distributions have the additional advantage that evidence for or against a model can typically accrue faster than when default priors are used (e.g., Stefan et al., 2019). This means that researchers are more likely to obtain strong evidence in favor of either model even when little data are available. In certain areas of psychology where data collection can be costly and sample sizes are typically small, this increased diagnosticity can be considered a major advantage of informed prior distributions.

It is important to note that prior specification in Bayesian modeling matters most when data are scarce. If there are ample data, the data will overwhelm the prior distribution (Vanpaemel, 2010; Wrinch \& Jeffreys, 1919). This means that posterior distributions derived from different prior distributions will be almost indistinguishable (note, however, that Bayes factors with different priors under the alternative model can still be different). The amount of data needed to overwhelm the prior distribution depends on the level of information contained in the prior distribution relative to the informativeness of the data. For example, some statisticians recommend weakly-informative prior distributions that include just enough subjective information to regularize and stabilize the model but can be quickly overwhelmed by the data (Gelman et al., 2008; Sarma \& Kay, 2020). This shows that "objective" and "subjective" prior distributions can be considered a continuum rather than a dichotomy. Prior distributions specified based on prior elicitation efforts from experts can lie anywhere along this continuum depending on the uncertainty of the experts and on the researchers' decision regarding the extent to which they want to incorporate the expert knowledge in their priors. 


\section{Prior Elicitation From Experts}

One principled way to specify an informed prior distribution is through prior elicitation from experts (O'Hagan et al., 2006). In contrast to other methods, for example prior specification based on the outcomes of previous studies (Verhagen \& Wagenmakers, 2014; Boehm et al., 2018), prior elicitation does not require existing data and is therefore widely applicable to almost all prior specification scenarios.

Prior elicitation techniques aim to transform qualitative plausibility judgments of field experts to probability distributions (O'Hagan et al., 2006). A prior elicitation procedure can best be described as a structured interviewing process where an interviewer, also called facilitator, guides one or more field experts through the process of formulating their knowledge about parameters in a probabilistic form (Winkler, 1967; Garthwaite et al., 2005). Typically, prior elicitations are conducted face-to-face, but online questionnaires or telephone interviews are possible as well (Johnson, Tomlinson, Hawker, Granton, \& Feldman, 2010). Several protocols have been developed that structure the elicitation procedure and intend to minimize cognitive biases, such as the availability or overconfidence bias or anchoring effects (O'Hagan, 2019). A common feature of all prior elicitation procedures is that they directly or indirectly ask experts to define a limited number of summary statistics of the resulting probability distribution, such as quantiles or a most likely value (O'Hagan \& Oakley, 2004). These summary statistics are then used in a second step to fit a probability distribution using a minimization algorithm (Morris et al., 2014). When multiple experts participate in the prior elicitation, the resulting distributions can be combined into a single prior distribution in a third step (Albert et al., 2012).

Prior elicitation from experts has been repeatedly proposed as a solution for prior specification in Bayesian modeling in psychology (Lee \& Vanpaemel, 2017; Lee et al., 2019; Chow \& Hoijtink, 2017; Dienes, 2019; Baldwin \& Fellingham, 2013; Etz \& Vandekerckhove, 2018; Depaoli, 2014). Although Bayesian inference has only recently gained attention in psychology (Vandekerckhove et al., 2018; Andrews \& Baguley, 2013; Tendeiro \& Kiers, 2019), prior elicitation efforts can already be found across several different psychological 
disciplines. For example, Gronau et al. (in press) used prior elicitation in an analysis of a replication of Strack et al.'s (1988) facial feedback study. Bolsinova et al. (2017) used an elicitation procedure to specify priors for the difference in difficulty of two psychological tests. In clinical applications, Mossman et al. (2015) conducted a prior elicitation for the base rate of feigning cognitive impairment in the Test of Memory Malingering, and AlAwadhi \& Garthwaite (1998) elicited priors for a multivariate normal model on intelligence and memory performance after head-injuries. Recently, Sarma \& Kay (2020) used prior elicitation to formulate a Bayesian model of the Balloon Analogous Risk Task (BART) that measures risk-taking behavior, and Tessler \& Goodman (2019) elicited beliefs about the prevalence of certain features (e.g., being female) in semantic categories.

There are several review articles on prior elicitation techniques (e.g., Garthwaite et al., 2005; Johnson, Tomlinson, Hawker, Granton, \& Feldman, 2010; Grigore et al., 2013) as well as two monographs covering probability elicitation in various research settings (O'Hagan et al., 2006; Dias et al., 2018). Although we believe that it is beyond the scope of the current article to provide a systematic review of all instances of prior elicitation being used within psychology, we provide a brief review of some specific examples in our online supplementary materials (https://osf.io/tbz6n/). In the remainder of this article, we will focus on generic practical decisions that researchers have to make in the process of prior elicitation when the goal is to find suitable priors for Bayesian parameter estimation or model comparison. We find that although current prior elicitation protocols are constructed to avoid cognitive bias (O'Hagan, 2019), they allow for considerable flexibility regarding these practical decisions which can, in turn, substantially influence the outcome of the elicitation procedure. In the following sections, we will investigate (1) decisions about the setup of the prior elicitation procedure; (2) decisions about the core elicitation process; and (3) decisions about the final distribution fitting, and discuss how these decisions can shape the outcomes of the elicitation process. Figure 2 gives an overview of the decisions that we will address in this paper. 


\begin{tabular}{|c|c|c|}
\hline Setup of the Prior Elicitation & Core Elicitation Process & $\begin{array}{l}\text { Combination of Priors from } \\
\text { Different Experts }\end{array}$ \\
\hline $\begin{array}{l}\text { - For which parameters should prior } \\
\text { distributions be elicited? }\end{array}$ & $\begin{array}{l}\text { - Which prior elicitation technique } \\
\text { should be chosen? }\end{array}$ & $\begin{array}{l}\text { - Should elicited priors be combined } \\
\text { to one distribution? }\end{array}$ \\
\hline $\begin{array}{l}\text { Who should be the experts in the } \\
\text { prior elicitation? }\end{array}$ & $\begin{array}{l}\text { - How should the prior elicitation } \\
\text { technique be implemented? }\end{array}$ & $\begin{array}{l}\text { - Should experts interact to find a } \\
\text { consensus distribution? }\end{array}$ \\
\hline $\begin{array}{l}\text { - How many experts should be } \\
\text { invited? }\end{array}$ & $\begin{array}{l}\text { What functional form should be } \\
\text { used to fit the prior? }\end{array}$ & $\begin{array}{l}\text { - Should priors be combined using } \\
\text { mathematical rules? }\end{array}$ \\
\hline $\begin{array}{l}\text { - How much common ground } \\
\text { should be established between the } \\
\text { experts before the elicitation? }\end{array}$ & $\begin{array}{l}\text { - Should experts be allowed to } \\
\text { adjust the prior? }\end{array}$ & \\
\hline
\end{tabular}

Figure 2. Overview of outcome-relevant decisions that researchers have to make in the prior elicitation process that are discussed in this paper.

\section{Decisions about the Setup of the Prior Elicitation}

Before the core elicitation session can take place, a researcher needs to make several basic decisions regarding the setup of the prior elicitation. For example, researchers need to decide for which model parameters they want to elicit prior distributions. These can be all model parameters, or only a subset of them. Furthermore, the experts for the elicitation need to be selected. Here, decisions have to be made such as who should be considered an expert, how many experts should be invited, and to what extent should experts diverge in their theoretical backgrounds. These decisions that take place before the core elicitation session are somewhat broader than decisions at later stages of the prior elicitation process and their implications on the elicitation results are more difficult to quantify. However, as will become clear in the next sections, they are equally important as they contribute to methodological flexibility and interact with decisions made later in the process.

\section{For Which Parameters Should Prior Distributions Be Elicited?}

Parameter selection for prior elicitation is especially challenging in the context of Bayesian model comparisons; with multiple models in place, a larger number of parameters needs to be taken into account and prior distributions for similar parameters in different models can typically not be considered completely independent of each other. In psycholog- 
ical research, the most common Bayesian model comparison is a Bayesian null hypothesis test that compares a null model and an alternative model (Wagenmakers et al., 2010). ${ }^{4}$ Null models usually set the parameter of interest (e.g., a mean in a $t$-test) to zero or another fixed value which makes a prior elicitation for this parameter unnecessary. Alternative models typically assign a prior distribution to the parameter of interest, which makes a prior elicitation for this parameter possible (Rouder et al., 2009; Sinharay \& Stern, 2002).

Typically, statistical models contain nuisance parameters that are not the focal quantity of the hypothesis test (R. L. Berger \& Boos, 1994). Nuisance parameters are parameters that are necessary for the specification of the problem, but are not by themselves subject of scientific interest (Liang \& Zeger, 1995). Even subjective Bayesians typically assign default prior distributions to nuisance parameters (J. Berger, 2006), for example Jeffreys' prior $\left(p\left(\sigma^{2}\right)=1 / \sigma^{2}\right)$ for the variance in a $t$-test (Gronau et al., in press). Thus, prior elicitation mostly focuses on prior distributions for the parameters of interest, although it would technically be possible to elicit priors for nuisance parameters as well (Dienes, 2008, p. 94). There are two apparent reasons for this unequal treatment of parameter groups. The most important reason is that priors on nuisance parameters tend not to influence the conclusions of Bayesian analyses which makes it questionable whether time and other resources should be dedicated to prior elicitation in these cases (Chaloner, 1996). Additionally, it is not clear whether experts are able to make reliable plausibility judgements for nuisance parameters. This makes it questionable whether using elicited priors on nuisance parameters would lead to better models (O'Hagan et al., 2006, p. 35).

There is a specific case in the practice of Bayesian model comparisons where the feasibility of expert elicitation for parameters of interest may be questionable, that is, when one model is clearly preferred over another by the (available) experts. As Garthwaite et al. (2005) state, in general, the objective of prior elicitation is "to express the expert's current knowledge in probabilistic form". However, when the current knowledge renders the assumptions of one model unlikely, deriving a realistic prior distribution for effect size under

\footnotetext{
${ }^{4}$ But note that the Bayesian paradigm allows you to compare any models you can specify (Etz et al., 2018; Evans \& Servant, 2020).
} 
this model might be difficult for field experts, as they have to selectively disregard their current knowledge about the low model probability in the elicitation process and focus on the (however unlikely) scenario that the model assumptions hold. For example, in Bayesian null hypothesis testing, field experts may entertain strong beliefs in favor of the null model even though researchers deem it probable that an effect could exist. In our experience, experts in this case tend to confuse their individual small prior model probability for the alternative model with a small expected effect size. For example, imagine health experts being asked for the most likely effect size of a new treatment for late stage Alzheimer's disease. As most treatments have been shown to be ineffective in earlier studies (Kumar et al., 2015), it is likely that none of the available experts believes in the existence of an effect. When pressured to specify a prior distribution under the alternative model, they are likely to use the heuristic "The effect is unlikely to be there, so if it exists, it must be very small". However, we argue that this heuristic can be misguided because the probability of the existence of an effect (i.e., "The new treatment is effective") does not have to be informative about the size of the effect given that the effect exists (i.e., "The expected health effect given that the new treatment works"). On a formal level, we expect that the experts run a risk to induce a correlation between the prior model probability, $p(\mathcal{M})$, and the prior distribution within the model, $p(\theta \mid \mathcal{M})$, if they assign a low prior probability to the model. We argue that this might render an unbiased elicitation difficult in this case.

To conclude, researchers need to carefully consider which prior distributions should be elicited from experts before the elicitation procedure starts. This decision is important because elicited informative priors might differ substantively from alternative default solutions. Eliciting prior distributions for all parameters might not be possible because of time constraints or because experts are likely to be unable to provide reliable non-biased plausibility assessments. Pre-elicitation interviews might help to identify what parameters are suitable for elicitation, for example by asking experts how confident they are with providing plausibility assessments for parameter values, but there is no evidence as to whether such interviews can provide reliable results. When elicitation is considered feasible but dif- 
ficult for the experts, both expert and facilitator training before the elicitation may help to mitigate biases and enable experts to provide more accurate and reliable distributions.

\section{Who Should Be the Experts in a Prior Elicitation?}

As stated above, prior elicitation requires the participation of one or more field experts. But what is an expert? According to Bolger (2018), candidates should display both substantive and normative expertise. Substantive expertise refers to the possession of knowledge about the concepts, theories, and state of literature in the research field in question. Normative expertise required for prior elicitation mainly refers to sufficient familiarity with the most important statistical concepts used for the elicitation, such as expressing uncertainty as probabilities and understanding the relevant parameters of the statistical model (O'Hagan et al., 2006, p. 27). Questionnaires such as the 'Expert-Skills Questionnaire' (European Food Safety Authority, 2014) can be used to screen experts before the elicitation procedure, but the concrete operationalization of the expert definition remains highly situation-specific and is left to the researcher.

In practice, the number of experts available for prior elicitation is often limited. In general, the more specialized the field of application is, the fewer field experts with sufficient substantive knowledge will be available. However, even if a large number of substantive experts exists, the complexity and parametrization of the statistical model can diminish the number of suitable experts. For example, more experts will be comfortable with making probability judgments about raw parameters (e.g., means) than about standardized parameters or hyperparameters in hierarchical models. The SHELF elicitation framework (O'Hagan \& Oakley, 2019) suggests to train experts in making the required probability judgments before the elicitation procedure starts. However, the maximum duration of these trainings is limited as most experts' willingness to participate will depend on the amount of time they need to commit to the elicitation procedure. For complex models, it is unlikely that all relevant information can be conveyed within the short time frame of these training sessions in a way that experts can confidently use the relevant statistics in the elicitation 
procedure.

To conclude, we argue that the presence of sufficient normative knowledge needs to be considered carefully in the selection of experts, as well as practical factors such as time constraints and available resources (European Food Safety Authority, 2014). The selection of experts can have considerable implications for the results of the prior elicitation because different experiences, theoretical convictions, levels of normative expertise, and even personality characteristics can prompt experts to provide different assessments in the prior elicitation process.

\section{The More the Merrier?}

The question of availability directly connects to the question of finding the optimal number of participating experts. In the current literature, there is no general agreement regarding the answer to this question. For example, O'Hagan (2019) recommends 4-8 experts, Aspinall (2010) recommends 8-15 experts, and Grigore et al. (2013) recommend 612 experts. It is important to note that all of these recommendations are based on practical experience and theoretical considerations rather than on experimental evidence.

Dating back to Kadane (1986), an often-mentioned goal for expert recruitment is that the expert panel should be representative for the community in the research field. Experts should possess a similar level of expertise while contributing different perspectives on the topic (Grigore et al., 2013). This has been shown to be a sensible approach in parameter estimation contexts (e.g., Aspinall, 2010), where a combined prior distribution from a diverse expert panel can represent the current uncertainty about a quantity within a field. However, the recruitment of experts from different schools of thought and the subsequent pooling of the experts' elicited priors could be criticized from a model comparison standpoint. As prior distributions are part of the specification of models, and models formalize substantive theories (Vanpaemel, 2010), lumping together priors from experts with different individual hypotheses can lead to models that do no longer precisely represent any single substantive theory. There are two ways to resolve this issue: Either a high-consensus panel can be 
recruited, where all experts are likely to follow the same underlying school of thought, and their prior distributions can be pooled. Alternatively, a diverse panel can be recruited where only the prior distributions from similar experts are later combined, so that in the end multiple models arise that represent the different schools of thought. We will address the combination of prior distributions later in this paper.

To conclude, the number and diversity of experts can influence the variability of prior distributions that result from the process. Researchers can manage this variability based on the goals of the statistical analysis.

\section{How Much Common Ground Should Be Established Before the Elicitation?}

Even natural high-consensus groups of experts, such as the members of one research team, can come to diverging results in the prior elicitation procedure. For example, in a Bayesian hypothesis testing framework, experts can disagree as to whether the alternative hypothesis to test should be one-sided or two-sided - that is, whether the prior distribution should be truncated - or whether certain values should be assigned a very low prior probability. Although a certain amount of variability between elicited distributions is natural and potentially desirable (if experts have different backgrounds), a subsequent aggregation of qualitatively different elicited prior distributions can result in a vague final prior distribution, or even worse, a final prior distribution that provides a poor reflection of individual experts' assessments of probability (Estes, 1956; Ratcliff, 1979). This makes it important to find a balance between unique contributions and a-priori consensus of experts. A-priori consensus can either be achieved through recruitment only (as described in the previous section) or through communication between experts before the elicitation procedure.

Figure 3 shows three theoretical scenarios for different levels of consensus between experts, and how establishing common ground before the elicitation influences the potential for variability between experts. In the left panel, no communication between experts took place before the prior elicitation. In this scenario, the resulting prior distributions can be highly divergent, including truncated, bimodal, and highly skewed distributions. The 

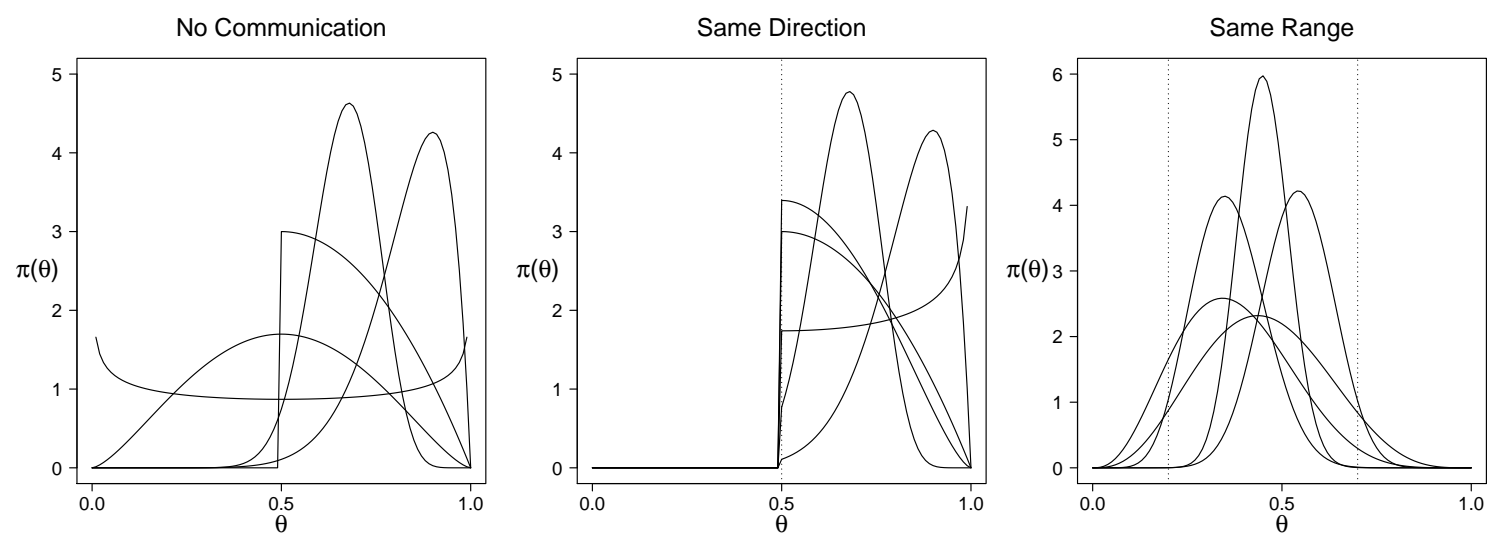

Figure 3. Hypothetical examples for elicited distributions with different levels of consensus between experts before the prior elicitation. All distributions are (truncated) beta distributions. In the middle panel, distributions are truncated at 0.5 to illustrate a scenario where experts agreed on the sidedness of the hypothesis before the elicitation. The right panel shows distributions where experts agreed before the elicitation that the most plausible parameter values lay between 0.2 and 0.7 .

middle panel shows a scenario where experts agree on the direction of the hypothesis prior to elicitation such that all distributions are truncated below a certain cutoff value (here: $\theta=0.5)$. As can be seen, the distributions are still divergent, but only within a limited range. Many elicitation methods (e.g., Morris et al., 2014) require experts to define a wide credible interval at the beginning of the elicitation procedure. The panel on the right shows a scenario where experts had to agree on this range of most plausible values before the elicitation. We depict the hypothetical scenario that the experts agreed on a range between 0.2 and 0.7. As can be seen, for all resulting prior distributions, the prior mass is mainly located within the bounds of the range of plausible parameters, which limits the discrepancy between the prior distributions.

As a general rule, the more constraints are imposed upon experts by consensus establishing procedures, the less distinctive input can be acquired from each expert. In the most extreme case, the complete elicitation procedure could be constructed as a group task where the immediate output is a single prior distribution (O'Hagan et al., 2006, p. 186f). However, classic social psychology research suggests that the elicitation results will be more subject to group dynamics as the amount of group activity within the elicitation 
procedure increases (European Food Safety Authority, 2014). For example, group settings might induce experts to suppress dissent and the consideration of alternative solutions due to phenomenons such as group-think (Janis, 1972) or social conformity (Asch, 1956). The group elicitation result might also be more influenced by individuals with certain personality characteristics or a higher attributed seniority (Bolger, 2018; Gosling, 2018). On the other hand, if an experienced facilitator can counteract these biases, single experts may profit from a group discussion by acquiring relevant knowledge (Reagan-Cirincione, 1994). Methods to structure group discussions are discussed later in the paper.

An alternative to establishing common ground before the elicitation is to treat experts as separate entities first (as in the left panel of Figure 3) and to pool priors that can reasonably be assumed to represent the same hypothesis in a second step. In this way, prior elicitation can act as a hypothesis-generating procedure in which multiple competing hypotheses prevalent in the scientific community can be extracted and instantiated as statistical models. Approaches such as hierarchical clustering analyses (e.g., Aggarwal \& Reddy, 2014) or latent mixture-model approaches (e.g., Navarro et al., 2006) could be used to achieve a statistically robust grouping of experts. However, a standard statistical procedure for grouping experts into clusters of qualitatively similar prior distributions has not yet been developed.

In conclusion, researchers engaging in prior elicitation must be aware that the rules they set for communication between the experts influence the resulting distributions. There is a clear trade-off between harvesting the largest possible amount of information from each expert (when communication is limited) and achieving converging results from multiple elicitation procedures (when experts agree on common ground beforehand). The desirable amount of communication before the elicitation procedure is likely to be dependent on later steps of the elicitation process and analyses, for example on whether the elicited distributions should be pooled or analyzed separately. 


\section{Decisions about the Core Elicitation Process}

After nominating the experts, selecting parameters, and setting the rules for communication between the experts, researchers need to shape the core elicitation process, that is, the details of the interview procedure from which prior distributions of experts are derived. All common structured elicitation methods are designed with the goal of minimizing noise arising from this interview procedure, such as interviewer effects or cognitive biases (Hanea et al., 2018; O’Hagan, 2019). However, many decisions about the elicitation procedure are still left to the researcher, both on the macro level (i.e., choice of an elicitation technique) and on the micro level (i.e., choices within an elicitation technique) and these decisions can influence the outcome of the elicitation process. Listing all possible elicitation techniques or providing a comprehensive account of the multitude of choices within a technique is beyond the scope of this paper. Therefore, the next sections will outline these decisions on a general level and provide several examples to showcase how they could influence the elicitation results. At the end of the elicitation procedure, a probability distribution needs to be fitted to the experts' elicitation results. In the following sections, we therefore also investigate how the facilitator's choice of a distributional family and the level of expert control over the final distribution can influence elicitation results.

\section{Which Prior Elicitation Technique?}

The current literature comprises a large variety of elicitation techniques (see Johnson, Tomlinson, Hawker, Granton, \& Feldman, 2010; Grigore et al., 2013; Garthwaite et al., 2005, for an overview). These range from very general methods that can be applied to a multitude of models (e.g., Morris et al., 2014) to very specific methods that are designed for a single model application (e.g., Chaloner et al., 1993).

Typically, prior elicitation techniques aim to assess quantiles of experts' inherent probability distributions on parameters (Grigore et al., 2013). The methods differ in the framing of the questions and in the number of elicited quantiles. For example, in some methods experts are asked to "bet" coins on parameter values while others ask for quantiles 
directly, and some methods elicit only 3 quantiles while others elicit 10 or more (Morris et al., 2014). One of the most popular prior elicitation techniques is the Bisection Method (Grigore et al., 2013). Here, experts first specify a value (the median) such that the parameter is equally likely to be less or greater than this value. Then, experts are told to assume that the parameter is, in fact, smaller/larger than the median and the lower/upper quartile are assessed in the same way as the median (O'Hagan et al., 2006, pp. 100f). In a similar manner, the Tertile Method asks the experts to specify the median as well as the 33rd and 66th percentile (Garthwaite \& O'Hagan, 2000). In contrast, the Complementary Intervals (Leal et al., 2007) and Probability Method (Morris et al., 2014) require experts to express their subjective probability that the parameter lies within certain predefined intervals. The Roulette Method (Johnson, Tomlinson, Hawker, Granton, Grosbein, \& Feldman, 2010) frames the elicitation as a betting game. Here, the facilitator gives a number of coins to the expert which the expert needs to "bet" on several parameter intervals. The number of coins per interval is then transformed into a probability statement. Similarly, the Histogram Technique (van Noortwijk et al., 1992) asks experts to adjust the bars of a histogram according to their subjective probability.

Most prior elicitation methods, like the ones mentioned earlier, involve questions about the model parameters themselves (e.g., Winkler, 1980; Grigore et al., 2013). However, it is also possible to elicit prior distributions indirectly by asking experts to answer questions about the plausibility of observable data patterns (Kadane, 1980). For example, the experts could indicate the plausibility of a certain number of successes in a binomial sample (Winkler, 1967) or the values of a criterion variable in a linear regression model (Winkler, 1980). The experts' predictions are then used to reconstruct the prior distribution that most closely matches these predictions, such as through the marginal likelihood formula in Equation 2. Importantly, indirect elicitation methods are designed to reduce the cognitive load on experts, thus making the elicitation procedure accessible for experts with less normative expertise (Bolger, 2018; Kadane, 1980).

However, it is important to note that in addition to the many sources of method- 
ological flexibility discussed within this article, indirect approaches to prior specification contain several unique limitations. Firstly, these approaches are even more model specific than those that directly query the parameter values, as reconstructing the prior distribution depends on the likelihood function (Winkler, 1980). Secondly, reconstructing unique prior distributions from the elicited data patterns becomes more difficult with increasing model complexity, as for models with highly correlated parameters different combinations of prior distributions can lead to similar predictions about observable data (Gutenkunst et al., 2007). This is especially noteworthy since indirect elicitation is often recommended for complex models for which it might be difficult to recruit experts with sufficient normative expertise (Kadane, 1980). Note that we base the examples within this section of the article on direct elicitation techniques, though the general points of discussion (see Figure 2) are still applicable to indirect approaches.

Even though there is some evidence that not all elicitation methods work equally well in practice (e.g., Winkler, 1967; Morris et al., 2014), only few studies have directly compared different elicitation methods in terms of their psychometric quality, comprehensibility, or efficiency (Winkler, 1967; Johnson, Tomlinson, Hawker, Granton, \& Feldman, 2010; Grigore et al., 2016). The few existing validation studies suffer from small sample sizes and are typically conducted in highly specialized application domains so that their generalizability is questionable (Grigore et al., 2016; Garthwaite \& O'Hagan, 2000). Therefore, little guidance exists for researchers regarding which elicitation technique they should choose. However, existing research on risk taking and decision making suggests that it is likely that the choice of the elicitation technique will have an influence on the functional form and validity of resulting distributions. For example, betting approaches might diminish overconfidence and thus result in wider distributions (Ferretti et al., 2016) or experts' underestimation of sampling variability (Tversky \& Kahneman, 1971) might lead to tighter prior distributions in indirect elicitation approaches. More research is needed, however, to gauge the effects that different elicitation methods exert on the elicitation results. 


\section{Choices Within an Elicitation Technique}

Although current protocols (e.g., Gosling, 2018) try to standardize prior elicitation, there are often almost as many "little choices" within an elicitation method as there are choices between elicitation methods. For example, the MATCH tool for prior elicitation (Morris et al., 2014) suggests that experts should always provide a range at the beginning of the elicitation session, that is, a wide credible interval of their prior distribution. This is often necessary for the purpose of graphical elicitation when the elicited quantity has to be drawn on an axis, as it is the case in the Roulette Method or the Histogram Technique. The definition of the range is not specified clearly by the MATCH authors, so it can either be left undefined ("Please specify a range of plausible values") or specified as a $90 \%, 95 \%, 99 \%$ or any other credible interval. Figure $4 \mathrm{~A}$ shows the impact of this choice on the resulting prior distributions in a Roulette Method elicitation under the assumption that the expert's coin allocation (see middle panel in Figure 4C) remains unchanged. Arguably, the differences between the distributions are not large. However, the range specification that is given to the experts may also interact with their level of confidence, that is, it could encourage experts to spread their coins more or less widely across the available parameter intervals (see Figure 4C for an example). Figure 4B shows how this could have additional influence on the resulting distributions for two different range specifications.

Another example regarding variability within an elicitation method is the number of coins and parameter intervals in the Roulette Method. O'Hagan \& Oakley (2019) recommend 10 to 12 intervals and 20 or 25 coins. The consequences of this choice can be seen in Figure 5. If an individual expert always follows the strategy to assign one coin to each interval and the remaining coins to the middle categories, the resulting prior distribution depends heavily on the number of coins the expert was given. The number of intervals does not influence the distribution as much, but the difference is still perceivable. Of course, in reality the expert might react to the changes in the number of coins and the number of intervals by distributing the coins in a different manner. However, the question remains how sensitive experts are to these subtle changes in the design of the elicitation. It also 
A

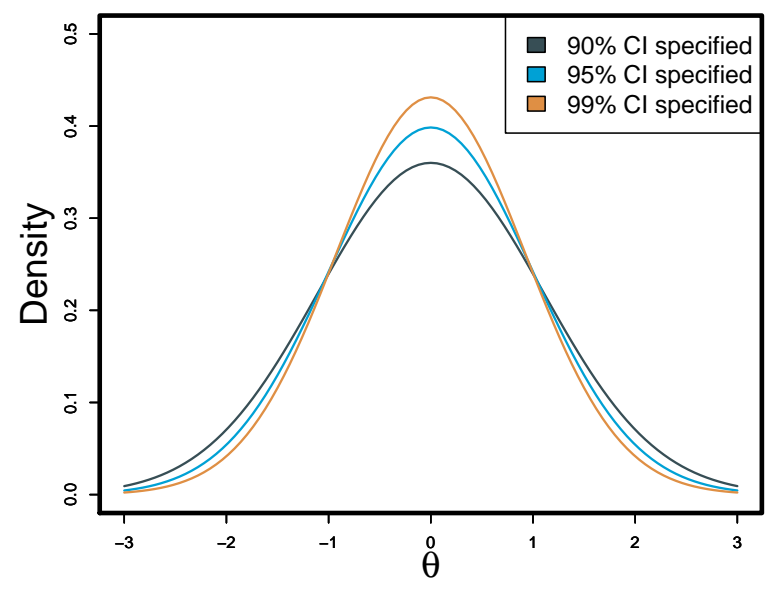

B

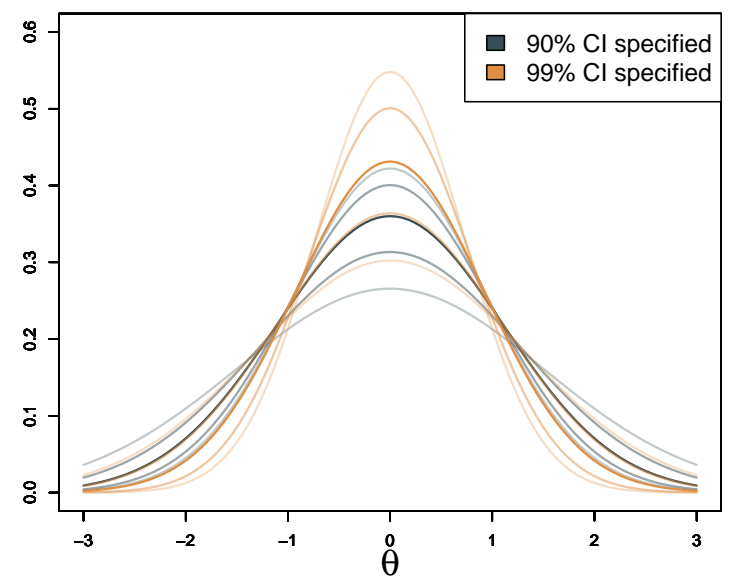

C
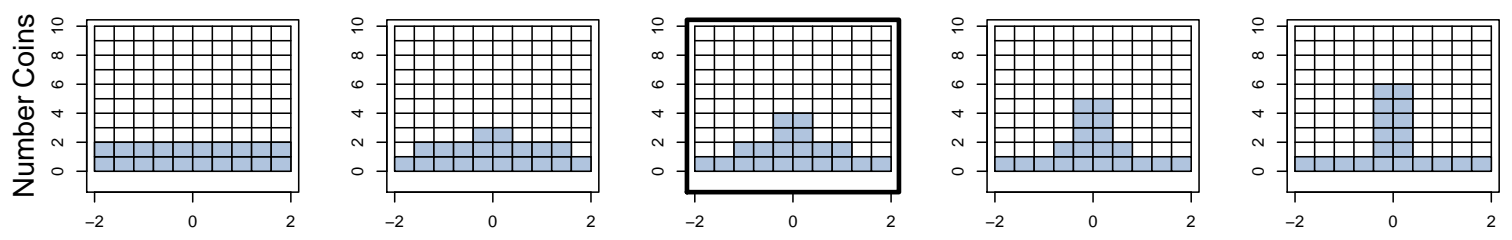

Figure 4. Panel A. Three prior distributions fitted to the same betting data in the Roulette Method based on three different definitions of the prior range. Panel B. Different prior distributions if prior range interacts with certainty as depicted in Panel C. Deviations from the certainty assumed in Panel A are depicted by increasingly lighter colors

. Panel C. Coin allocations depicting increasing certainty. The middle coin allocation was used in Panel A.

becomes clear from Figure 5 that, if an initial credible range is set, the number of available coins limits the steepness of the resulting prior distribution. Generally, more excess in the distribution is possible if more coins are available. Additionally, the choice of certain combinations of coins and intervals forces experts towards certain distributions. For example, when 25 coins have to be distributed on 10 intervals, a symmetric distribution is not possible. This may not only be uncomfortable for experts who are no longer able to express their inherent probabilities properly, but also nudge experts towards formulating non-symmetric distributions.

In practice, it is difficult to predict the effects of small changes in elicitation protocols on the resulting prior distributions. However, conjointly, they constitute a high amount of flexibility in the prior elicitation process that has been largely neglected in the current 


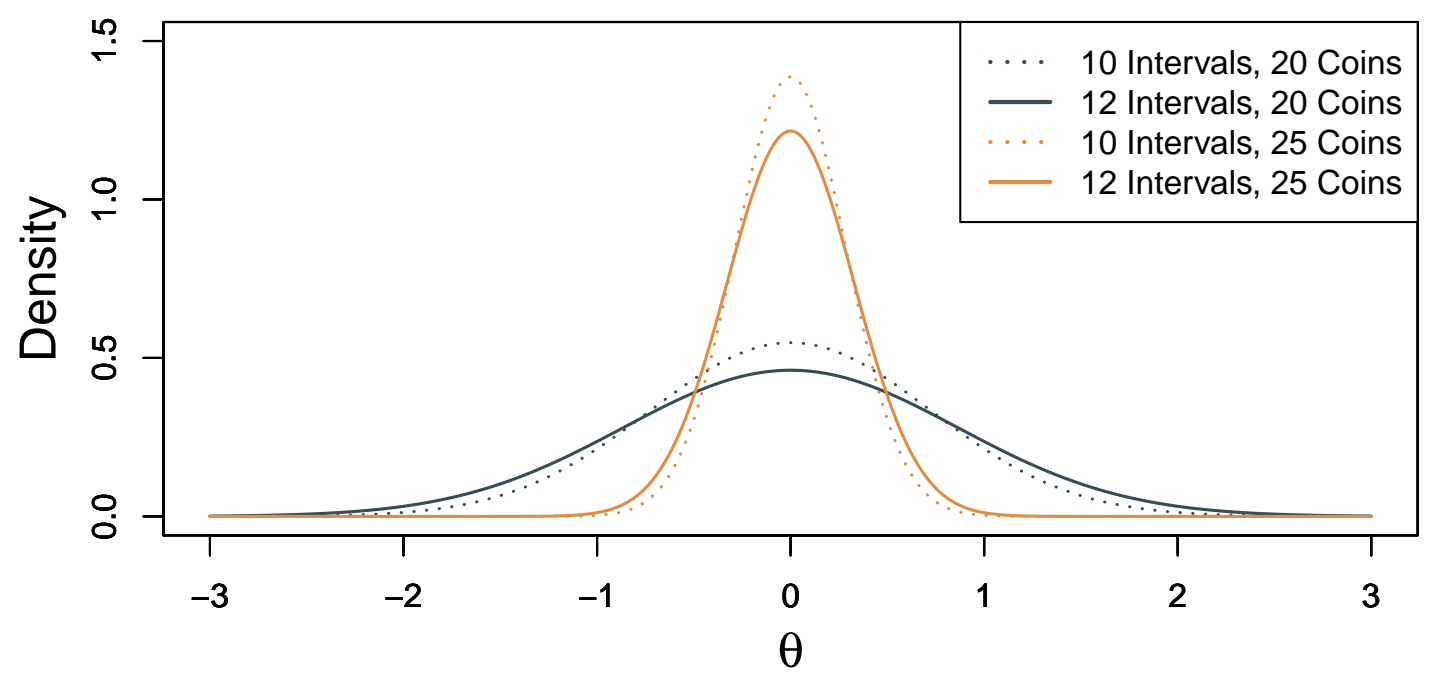

Figure 5. Resulting prior distributions for an elicitation method with 10 or 12 intervals and 20 or 25 coins for an individual expert who follows the same strategy: Assign one coin to each interval and the remaining coins to the two middle intervals.

literature. More research is needed to understand the implications of these decisions and to reach a consensus about potential best practices.

\section{What Functional Form Should the Prior Have?}

It is important to note that prior elicitation techniques require the facilitator and/or the expert to make additional assumptions about the elicited distributions. For example, three elicited quantiles such as in the Bisection Method are not sufficient to fully determine a prior distribution when more than one distributional family is considered. Figure 6 illustrates this by showing three distributions that share the same quartiles and could therefore be fitted to the same elicitation results. These distributions do not only differ distinctly in their shape but also reflect qualitatively different beliefs. For example, whereas $\theta=0$ is the most likely value in the normal distribution, it is considered very unlikely in the normal mixture, and while the normal and normal mixture distribution assign some (low) probability to extreme values, these are logically excluded under the uniform distribution. 


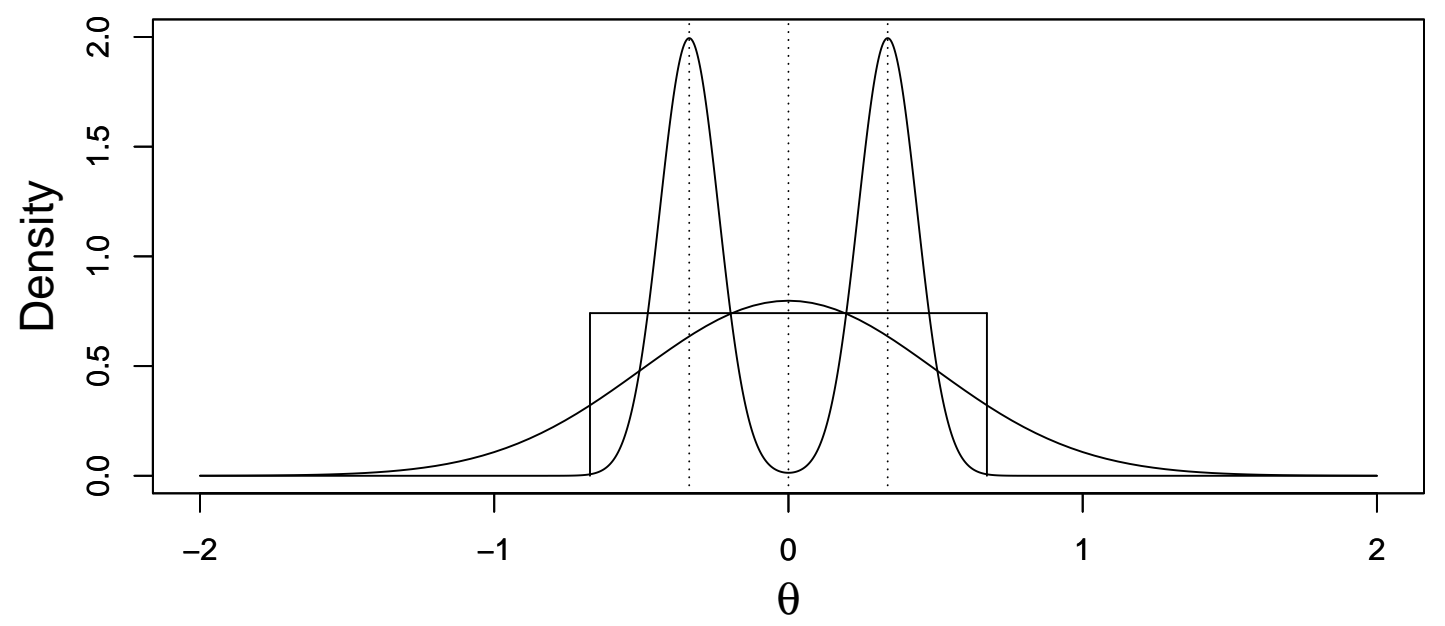

Figure 6. Three distributions with the same quartiles: Normal(0, 0.5), Uniform(-0.337, $0.337)$, Normal Mixture $\left(\mu_{1}=-0.337, \mu_{2}=0.337, \sigma_{1}=\sigma_{2}=0.1\right)$. Dotted lines show the location of the quartiles.

The uncertainty about the shape of the distribution could be partially resolved by obtaining more information from experts (i.e., elicit more quantiles). However, this comes with other limitations, for example, a higher cognitive load and a longer duration of the elicitation procedure when quantiles are elicited separately.

The decision of which distributional family should be used is typically left to the researcher and, as shown before, can have severe consequences on the shape of the distribution. Often, the choice of a distributional family is not completely arbitrary. For example, conjugate priors provide computationally convenient solutions for a multitude of Bayesian models (Fink, 1997) and are therefore often preferred in practice. However, increasing computational power and modern MCMC sampling methods (e.g., Evans \& Annis, 2019; van Ravenzwaaij et al., 2016) make it possible to use almost every prior distribution, and hence there are few technical restrictions as to which prior distribution families can be used. Of course, the chosen prior distribution needs to fulfill basic logical criteria, for example a prior on variance should be bounded to positive values. However, these logical criteria often do 
not greatly restrict the choice of available distribution families.

The above considerations make it clear that the choice of the distributional family can impact the fitted elicited distribution. This suggests that participating experts should be involved as much as possible in the creation of the final distribution to avoid misrepresentations of experts' probability assessments. When different distributions that vastly differ in shape can be fit equally well to the elicited quantiles, it is unlikely that all of them will accurately represent the expert's beliefs. We argue that the facilitator should be aware of these ambiguities and if possible resolve them by asking additional questions in the elicitation process or by letting the expert choose from different fitted distributions.

\section{Should Experts Be Allowed to Adjust the Prior?}

O'Hagan et al. (2006) claim that "feedback encourages an expert to think carefully about her assessments and [...] it can highlight apparent errors, thus providing an opportunity for the expert to correct them" (p. 176). While it is a largely uncontested view that reconciling logical inconsistencies through discussion during the elicitation is a reasonable approach (but see Osherson et al., 1997), it is much less clear whether the probability distribution that was fitted to the elicitation results should be discussed with the experts. For example, the MATCH elicitation tool (Morris et al., 2014) encourages this behavior by integrating the distribution fit within the elicitation procedure. Several families of probability distributions are fitted to the elicitation results and experts get the opportunity to choose a distributional family. Elicitation results (e.g., quartiles) can be retrospectively changed if the fitted distribution does not agree to the experts' probability assessments. More flexible procedures even make it possible to adjust single parameters of the resulting distribution (Gronau et al., in press).

On a higher level, the decision of whether or not to let experts adjust the resulting prior distribution boils down to the question of confidence in the elicitation procedure. The more retrospective adjustments are allowed based on the inspection of the resulting prior distribution, the higher is the chance to override the outcomes of the carefully planned 
elicitation procedure by an ad-hoc probability distribution sketching. On the other hand, adjusting the final distribution might give experts the opportunity to correct potential errors they made in the first part of the elicitation. Thus, it remains a choice between the devil and the deep blue sea.

If a researcher decides to let experts adjust their prior distribution, the question remains of how to deal with the fact that two distributions result from the process. Of course, for the sake of simplicity the researcher can decide to use the adjusted distribution and to discard the original distribution. However, there can be instances where it is not clear which distribution should be trusted more, for example, because the elicitation method was constructed to avoid biases that can be re-introduced through the adjustment of the distribution. In these cases it would also be an option to combine the two distributions to arrive at a single distribution in the end. The next section of this paper discusses different methods of combining distributions across experts. Most of these could also be used to combine distributions elicited with different methods from one expert.

To conclude, elicited prior distributions may change considerably if researchers allow experts to adjust them after the elicitation procedure. It is unclear how researchers should address the fact that two distributions, an original and an adjusted distribution, can result from the elicitation process. This gives rise to further flexibility in the elicitation process as there are multiple equally justifiable ways to handle the multiplicity of results.

\section{(How) Should Prior Distributions Be Combined?}

When a prior elicitation procedure is conducted independently with multiple experts, it results in at least as many prior distributions as there are experts. In rare cases, several experts may arrive at almost exactly the same distribution, but most of the time (especially when experts come from different backgrounds) there will be some variability in the resulting distributions. As Bayesian models require only one prior distribution per parameter, the researcher needs to decide which prior distribution(s) should be used in the final Bayesian modelling step. 
We argue that there are two qualitatively different options to deal with the multiplicity of prior distributions after elicitation. The first option is to handle all distributions separately, that is, introduce them individually in Bayesian analyses. We call this option the "No Pooling" option. The second option is to combine the prior distributions to a single prior distribution that can be used in Bayesian modelling. This can either be achieved via Behavioral Aggregation, that is, through interaction between the experts, or via Mathematical Aggregation, that is, through the application of formal rules (Wilson \& Farrow, 2018). In the following sections, we will detail the consequences and underlying assumptions of No Pooling, Behavioral Aggregation, and Mathematical Aggregation.

\section{No Pooling}

Although many elicitation frameworks implicitly assume or directly recommend the pooling of resulting prior distributions (Dias et al., 2018), we argue that it can also be informative to assess the distributions separately. As mentioned earlier, expert panels are often compiled with the goal to represent a broad spectrum of perspectives, experiences, or professional backgrounds (Kadane, 1986; Grigore et al., 2013). In this case, each expert can be seen as an individual entity representing a distinct school of thought. Following this argumentation, pooling experts would lead to a single prior that comprises different hypotheses, which might be theoretically hard to justify. Additionally, the combination of prior distributions of highly diverse experts may result in a very wide prior that provides little information. Using such a wide prior distribution counteracts one of the major goals of prior elicitation: to provide informed prior distributions that, when integrated into a model, represent a specific theory.

Instead of combining experts' distributions, researchers can opt to integrate each expert's prior distribution separately in the Bayesian analysis workflow. From a practical perspective, one way to achieve this is a prior sensitivity analysis, that is, the repeated computation of Bayesian analyses using different prior distributions. Typically, prior sensitivity analyses are conducted to explore the dependence of Bayesian analysis results on the choice 
of the prior distribution (Vanpaemel, 2010). A common critique of sensitivity analyses is that they become computationally unfeasible when models involve too many parameters (Bornn et al., 2010). Conducting a prior sensitivity analysis with the results from prior elicitation counteracts this critique by providing a limited number of theoretically justifiable prior distributions for which the sensitivity of analysis results can be assessed. Another way to integrate multiple resulting prior distributions in Bayesian analyses is to compare the resulting "expert models" to each other in terms of their predictive adequacy (e.g., by computing Bayes factors between them). If experts are representative for their respective fields, model comparisons between the expert models can provide additional substance to debates between expert communities. Alternatively, researchers can use Bayesian Model Averaging (e.g., Hoeting et al., 1999) to arrive at a balanced singular Bayesian analysis result that takes all experts into account. Compared to mathematical approaches that directly combine experts' distributions, Bayesian Model Averaging has the advantage to provide a data-dependent inherent weighting mechanism, that is, all individual expert models are weighted by their ability to predict the data. However, initial prior model probabilities have to be determined which can be challenging and provides yet another source of flexibility (Fragoso et al., 2018).

\section{Behavioral Aggregation}

If communication between experts is possible, an intuitive way of resolving discrepancies in experts' prior distributions is by finding agreement through discussion. As stated before, group discussions are at risk of being subject to biases such as group-think (Janis, 1972), social conformity (Asch, 1956), or group polarization (Lamm, 1988; for an overview, see Montibeller \& von Winterfeldt, 2015). However, a well-structured group discussion led by an experienced facilitator who is aware of these biases can outperform the accuracy of individual experts (Reagan-Cirincione, 1994). Additionally, behavioral aggregation approaches have the advantage that the resulting distribution is a clear consensus prior on which all available experts agree. As described later, this is not the case for mathematical 
aggregation where the result is an arithmetic combination of the single distributions and may not represent any expert's opinion (O'Hagan et al., 2006).

There are several protocols for behavioral aggregation of prior distributions that try to create a favorable environment in which the benefits of the group can be used and the risk of bias mitigated. One popular framework is the Delphi method (Dalkey \& Helmer, 1963). In this method, experts first anonymously share their prior distribution together with a justification, then they update their beliefs in the light of the other experts' justifications. This process is repeated until consensus is reached. The procedure relies on the idea of "better experts being able to explain their views more cogently" (O'Hagan et al., 2006, p. 189) and that biases are less prevalent when experts remain anonymous. Several other protocols, such as the IDEA protocol (Hanea et al., 2018) or the Nominal Group Technique (Delbecq et al., 1976), are based on the Delphi method. The SHELF elicitation framework proposes a behavioral aggregation procedure based on the notion of a "Rational Impartial Observer" (RIO). Here, the goal of the aggregation is not that experts convince each other of their respective priors, but that they evaluate their results from the perspective of a neutral external observer and create a distribution that would reflect this observer's state of knowledge (O’Hagan \& Oakley, 2019). Another aggregation method by DeGroot (1974) proposes to let experts assign weights to each other's distribution, let a facilitator combine the distributions with a linear combination function, report the resulting distribution to the experts and let them adjust their weights in an iterative process.

Even though behavioral aggregation methods provide an intuitively appealing way to combine experts' prior distributions, there are several caveats that researchers have to be aware of. First, we cannot expect any method to completely extinguish group-related bias from the process. Second, the level to which biases can be mitigated will depend largely on the moderator skills of the facilitator (Reagan-Cirincione, 1994). This also means that a biased facilitator can influence the results of the elicitation effort in their favor (Boyd \& Westfall, 1970). Additionally, if there is considerable disagreement and direct interaction between the experts, the reached level of consensus will also likely be dependent on the 
ability of the facilitator to lead the discussion (Herrera et al., 1996). Third, the choice of the concrete behavioral aggregation method influences the interpretation of the resulting prior distribution. For example, the resulting prior distribution can either be a true consensus prior (such as in the Delphi method) or a prior that is considered a rational "joint prior" by the experts as in the SHELF framework (which does not have to imply consensus). Fourth, researchers employing behavioral aggregation methods need to have procedures in place for the eventuality that no consensus can be reached. O'Hagan et al. (2006) propose a mathematical aggregation of the remaining prior distributions, but other options would be possible as well, for example a voting procedure within the behavioral aggregation or a No Pooling approach for the remaining prior distributions (in the case that the desired outcome does not have to be a single prior distribution; see previous section). Therefore, we conclude that the results of the elicitation procedure as well as their quality (i.e., reliability and biasedness) and interpretation depend highly on the specifics of the behavioral aggregation procedure.

\section{Mathematical Aggregation}

An alternative to behavioral aggregation approaches is the mathematical aggregation of prior distributions resulting from prior elicitations with multiple experts. In mathematical aggregation, the prior distribution functions are combined following a mathematical rule. Unlike behavioral approaches, mathematical aggregation does not suffer from the risk of inducing group biases. However, the resulting prior distribution may not represent a consensus between experts either.

Many mathematical aggregation methods have been proposed and providing a comprehensive review is beyond the scope of this article (for an overview, see Wilson \& Farrow, 2018). Here we discuss three fundamentally different classes of mathematical aggregation approaches: Linear Pooling, Logarithmic Pooling, and Supra-Bayesian Pooling (Genest \& Zidek, 1986; Albert et al., 2012).

In linear pooling methods, the aggregated prior distribution is equivalent to a 
(weighted) average of the individual experts' distributions (Wilson \& Farrow, 2018),

$$
f(\theta)=\sum_{i=1}^{E} w_{i} f_{i}(\theta)
$$

where $w_{i}$ is the weight given to each expert and $f_{i}(\theta)$ is each expert's individual prior distribution on the parameter $\theta$, and the weights have to be chosen such that $\sum_{i=1}^{E} w_{i}=1$.

In logarithmic pooling methods, the individual experts' distributions are combined with a multiplicative mixture instead,

$$
f(\theta)=k \prod_{i=1}^{E} f_{i}(\theta)^{w_{i}},
$$

where the weights $w_{i}$ are again constrained to sum up to one and the factor $k$ is a normalizing constant.

A central task for both the linear and the logarithmic pooling method is to determine the weights $w_{i}$. Clearly, the simplest solution is to choose equal weights. However, many other methods exist to determine the weights, for example based on the experts' performance on calibration questions, based on the importance a researcher or facilitator assigns to the experts, or based on experts' ratings of each others competence (Wilson \& Farrow, 2018).

Both the choice of the pooling method and the choice of the weights have a substantive influence on the resulting prior distribution. Figure 7 gives an impression of how much the resulting prior depends on these choices. Notably, the resulting distribution in the logarithmic pooling method is always unimodal (Rufo et al., 2012) while the linear pooling method typically results in multimodal distributions. Additionally, as can be seen in the bottom right panel of Figure 7, the combined prior distribution resulting from logarithmic pooling is zero for all parameter values for which at least one prior distribution is zero. On a conceptual level, this can represent the idea that parameter values that have been logically excluded by one expert should be rejected by all other experts as well.

Another method to combine prior distributions that differs distinctively from linear or logarithmic pooling is supra-Bayesian pooling (Albert et al., 2012; Gelfand et al., 1995). 
Prior Distributions from 3 Experts

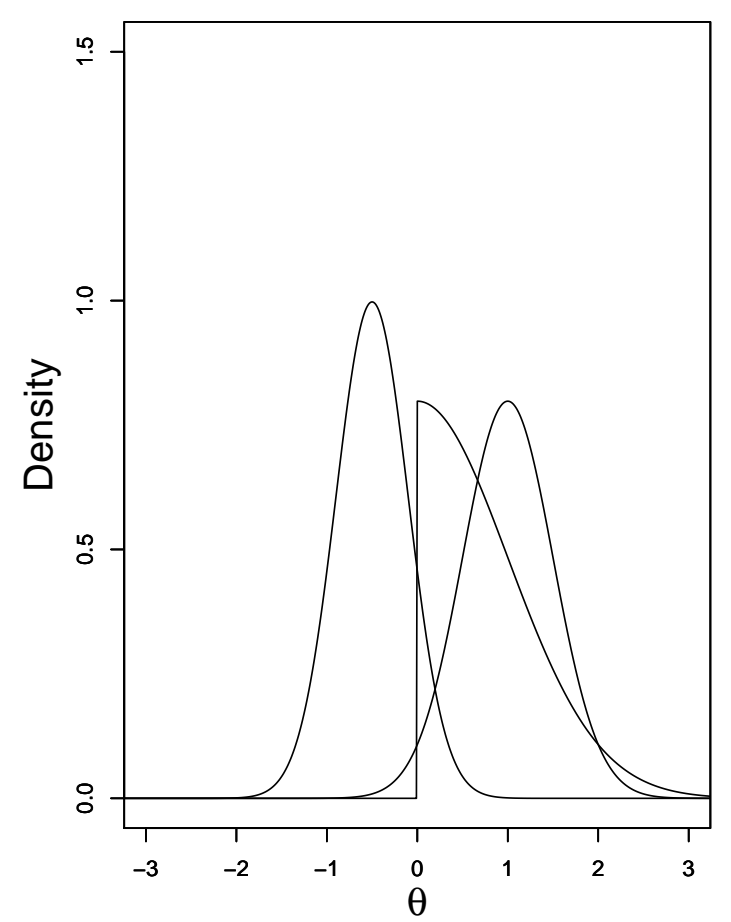

Linear Pooling

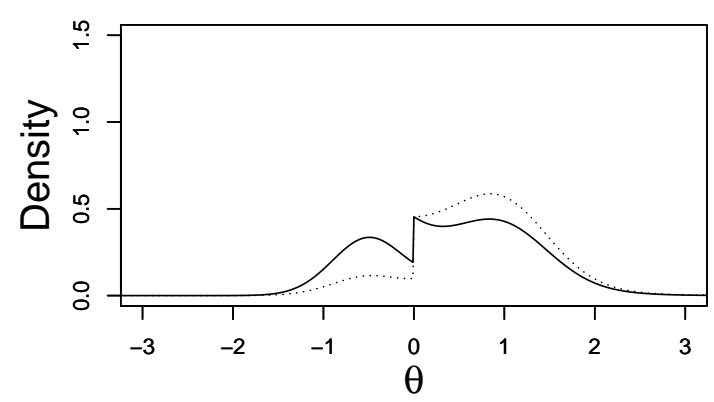

Logarithmic Pooling

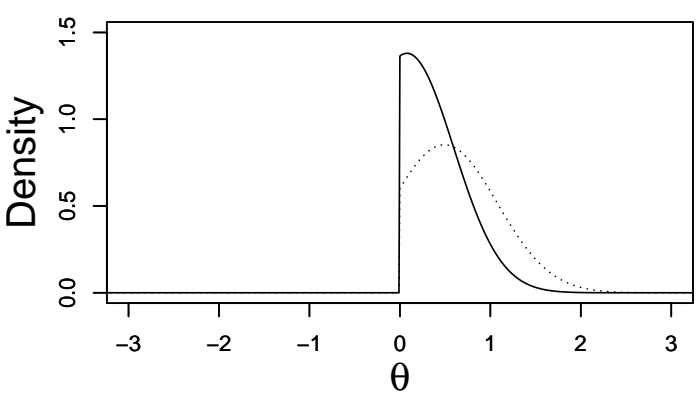

Figure 7. Left side: Three prior distributions to be combined $\left(f_{1}=\operatorname{Normal}(-0.5,0.4), f_{2}=\right.$ $\operatorname{Normal}(0,1)$ truncated at $\left.0, f_{3}=N(1,0.5)\right)$. Right side: Combination of the distributions via linear pooling and logarithmic pooling. Solid line shows pooling with equal weights, dotted line shows pooling where the $f_{2}$ and $f_{3}$ are weighed 4 times more than $f_{1}$.

Here, the idea is to view the elicitation results as data which can be used to update beliefs about the parameters of a combined prior distribution. For example, assume normal distributions were elicited from three experts with respect to a parameter $\theta$ (see top part of Figure 8). Each of these prior distributions can be described by a mean and a standard deviation, which we will consider as elicited data $x_{\mu i}$ and $x_{\sigma i}$, where $i$ is the index of the expert. ${ }^{5}$ Assuming that all experts come from the same population, each expert's $x_{\mu i}$ and $x_{\sigma i}$ should be drawn from common group-level distributions. For example, $x_{\mu i}$ could be drawn from a normal distribution with mean parameter $\phi_{\mu}$ and standard deviation parameter $\phi_{S D}$, and $x_{\sigma i}$ could be drawn from a gamma distribution with scale parameter $\phi_{s}$ and

\footnotetext{
${ }^{5}$ Note that other outcomes of the elicitation process, for example matching quantiles and probabilities, could also be considered as an input, but this would require a density function to be defined for these summary statistics.
} 


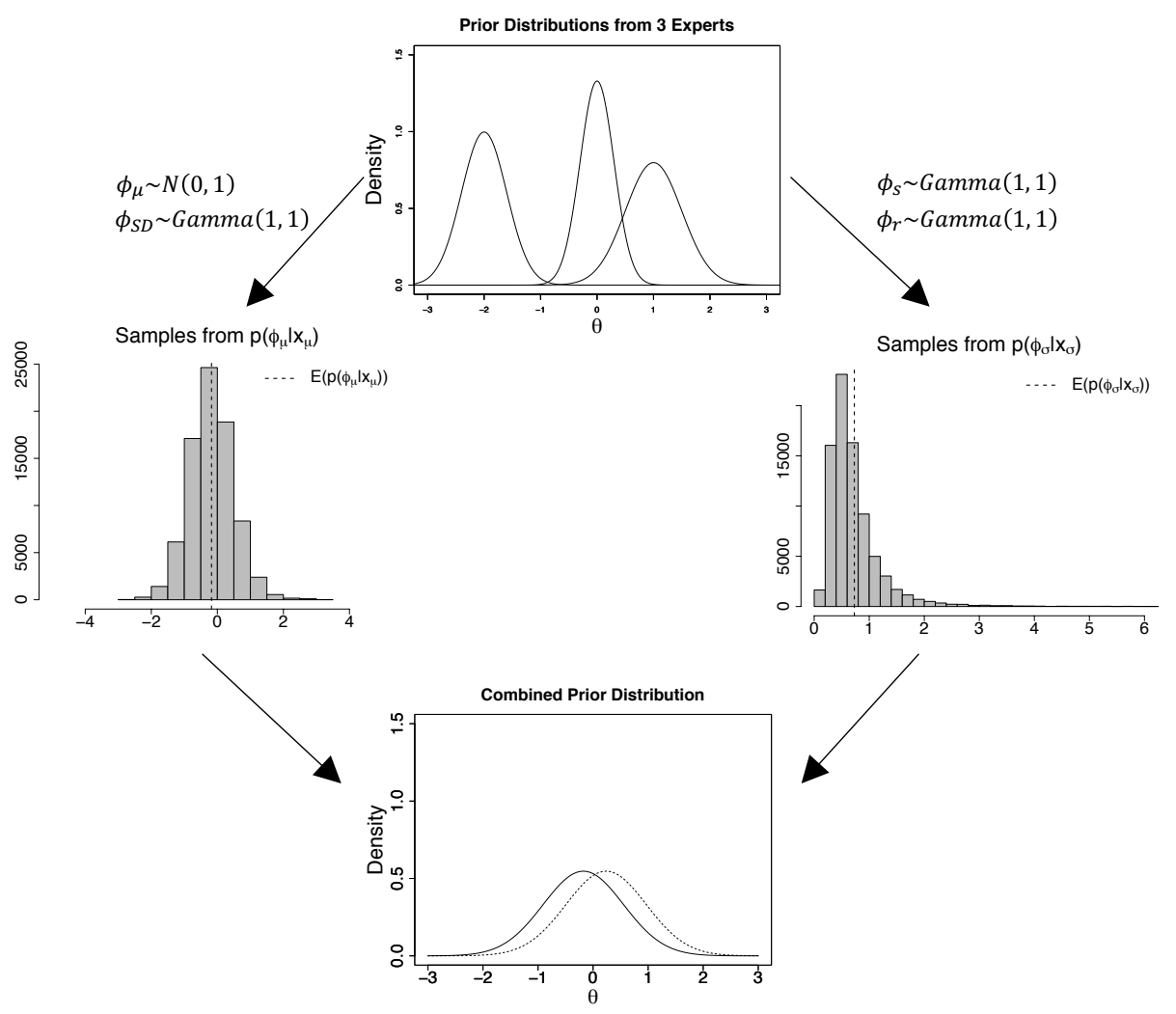

Figure 8. Updating parameters of a combined prior distribution with supra-Bayesian pooling. The dotted line in the lowest panel shows an alternative solution where the the prior on $\phi_{\mu}$ is defined as $\phi_{\mu} \sim N(1,1)$.

rate parameter $\phi_{r}$ whose mean can be described by $\phi_{\sigma}=\phi_{s} / \phi_{r}$. The supra-Bayesian model requires priors on $\phi_{\mu}, \phi_{S D}, \phi_{s}$, and $\phi_{r}$ which can be updated using the elicited data. The focal parameters in the model are $\phi_{\mu}$, which gives an estimate for the mean of the common prior distribution, and $\phi_{\sigma}$, which gives an estimate for the standard deviation of the common prior distribution. Posterior distributions for these parameters can be estimated using an MCMC sampling algorithm (see middle part of Figure 8). An estimate for the mean and standard deviation of a common prior distribution across experts can then be computed by determining the expected value of the posterior distributions of $\phi_{\mu}$ and $\phi_{\sigma}$ (see dotted lines in the middle of Figure 8 and the resulting common prior distribution on the bottom).

An advantage of the supra-Bayesian approach is that the supra-Bayesian model can 
be defined such that resulting common prior distribution comes from a distributional family that is easy to use later on. For example, in the model described above, the resulting prior distribution is always a normal distribution that can be used in a wide range of application contexts. Another advantage of the supra-Bayesian approach is that it provides the decision maker, that is, the researcher setting up the Bayesian model, with additional information about the elicitation process. For example, the width of the posterior distributions of grouplevel parameters (e.g., the $95 \%$ credible interval of $p\left(\phi_{\mu} \mid x_{\mu}\right)$ ) can be seen as a measure of uncertainty about the parameters of the elicited prior distribution, and the dispersion parameters of the group-level distributions (e.g., $\phi_{S D}$ ) can be interpreted as a measure of disagreement between experts about the prior distribution parameter.

However, the supra-Bayesian approach also has some limitations. The most pressing of these is arguably the dependence of the resulting prior distribution parameters on the group-level prior distributions in the supra-Bayesian model. As typically only few experts $(\mathrm{N}<20)$ participate in the elicitation, only few data points are collected that could inform the parameters of a shared prior distribution. Therefore, both the posteriors on the model parameters and the point estimates of the prior distribution parameters that are derived from them are highly dependent on the choice of the priors in the supra-Bayesian model. This can also be seen in the bottom panel of Figure 8. Here, two prior distributions are plotted that rely on the same data and where only one parameter in the prior on $\phi_{\mu}$ is changed in the supra-Bayesian model (from $\phi_{\mu} \sim N(0,1)$ to $\phi_{\mu} \sim N(1,1)$ ). Even this comparatively small change in the priors of the supra-Bayesian model leads to a visible change in the resulting combined distribution. The supra-Bayesian approach thus shifts the problem of the specification of prior distributions to a higher level as the question remains how to specify the prior distributions of the supra-Bayesian model. Another limitation is that the supra-Bayesian approach implicitly assumes that experts' knowledge can be reasonably represented by a single family of probability distributions (e.g., normal distributions as in the example above) and that it is not clear whether this assumption can hold up in practice. More advanced techniques like transdimensional MCMC methods would theoretically make 
it possible to find the best-fitting family of distributions and estimate the parameters of the combined distribution within each family of distributions at the same time, but given the small sample sizes, there will still be considerable uncertainty as to which distributional family can best capture the experts' knowledge.

As illustrated above, different mathematical aggregation approaches result in distinctly different prior distributions. It is important to acknowledge this flexibility because it allows decision makers to override experts' opinions relatively easily by the choice of the aggregation method. However, different mathematical aggregation methods follow different underlying logical principles, which can potentially guide the choice of method in different environments. For example, logarithmic pooling assumes that one expert can logically exclude parameter values for the whole resulting prior distribution while linear pooling and supra-Bayesian pooling do not give one expert this power. Supra-Bayesian pooling, on the other hand, emphasizes the role of the decision maker much more than unweighted linear or logarithmic pooling, such that it can be seen as a process to simply update already existing current knowledge based on expert opinions instead of creating a "new" prior distribution. Additionally, if a researcher decides to use unequal weights in linear and logarithmic pooling, this typically implies that the "quality" of experts can be directly measured (e.g., by calibration questions). In general, the assumptions underlying specific mathematical aggregation methods may be reasonable in some cases, but not be sensible in others. For example, in a typical hypothesis testing situation, it is questionable whether the knowledge of experts about the scenario at hand can be estimated from their knowledge about alternative scenarios and whether it is therefore sensible to use calibration questions to determine weights for linear or logarithmic pooling. We therefore argue that when researchers make the decision which mathematical aggregation method they want to use, they need to think carefully about the underlying assumptions.

One theme common to all mathematical aggregation approaches discussed in this section is that they combine the elicitation results on the level of the prior distribution. An alternative would be to combine elicitation results on the level of the model using Bayesian 
Model Averaging, as has been discussed earlier (Hoeting et al., 1999). Conceptually, this approach can be localized between "No Pooling" and "Pooling" methods, as although it results in a single Bayesian analysis result, experts' priors are not combined in one model. Bayesian Model Averaging is closely related to linear pooling methods because both methods provide a linear combination of the elicitation results. However, while the weights can be determined independent of the data in linear pooling, they are defined as the Bayesian posterior model probability in Bayesian model averaging (Hinne et al., 2019). Bayesian Model Averaging is also related to supra-Bayesian pooling as both methods provide an inherently Bayesian way to combine prior elicitation results. However, Bayesian Model Averaging does not make assumptions about relations between the experts' prior beliefs (e.g., that the priors elicited from experts are noisy representations of a common underlying prior distribution).

In sum, the use of mathematical aggregation methods enables researchers to combine the results of prior elicitations from multiple experts independent of group processes. However, they also allow for considerable flexibility in determining the aggregated prior distribution. This flexibility can partly be limited by the justifiability of aggregation method assumptions in specific research contexts. However, we argue that transparency about the aggregation process is nevertheless crucial because even within one aggregation method (e.g., the supra-Bayesian method), there are still numerous choices to be made that can greatly influence the outcome of the aggregation process.

\section{Navigating Practical Decisions in Prior Elicitation}

In the previous sections, we demonstrated that considerable methodological flexibility exists in prior elicitation. However, the issue of methodological flexibility or researcher degrees of freedom is not unique to prior elicitation. Indeed, researchers are constantly confronted with methodological decisions and conciously navigating these decisions can be seen as part of a researcher's expertise (Wicherts et al., 2016). Methodological flexibility only becomes problematic when researchers are not aware that they are making decisions 
that could influence their research results, when they cannot make well-informed decisions due to a lack of evidence, when they do not document their decisions adequately, or when they are incentivized to abuse the existing flexibility to tweak their research results (Head et al., 2015). Here, we mostly concentrated on raising awareness for the decisions that are implicit in the process of prior elicitation and for the potential consequences these decisions can have. This is a first step to enable researchers to carefully plan the prior elicitation process, consider the implications of alternative decisions, and be transparent about the prior elicitation process. In the following, we will discuss several steps that could be taken to improve transparency about decisions in the prior elicitation process, and provide several preliminary recommendations for prior elicitation in practice (cb. the section Preliminary Practical Recommendations for Prior Elicitation).

\section{Improving Transparency through Open Science Practices}

Similar to regular psychological experiments, we believe that thinking clearly and being transparent about methodological flexibility can greatly increase the credibility and methodological rigor of prior elicitation. As issues like publication bias or cherry picking of results might also arise in prior elicitation (Munafò et al., 2017), we believe that it would be prudent to consider applying Open Science practices, such as preregistration, open materials, or open data to prior elicitation procedures. Even though the application of Open Science practices to Bayesian modeling has raised some controversy in the past (Lee et al., 2019), there have been promising developments in this direction (e.g., Crüwell et al., 2019; Crüwell \& Evans, 2019) so we are confident that the application to prior elicitation would be both beneficial and feasible.

\section{Demonstrating the Impact of Decisions through Sensitivity Analyses}

One important lesson from the demonstration of methodological flexibility is that the outcome of a prior elicitation process cannot be interpreted as the single 'built-in' prior distribution of an expert. Rather, elicited prior distributions are also dependent on the 
prior elicitation process itself, which means that inspecting multiple potential outcomes can create interesting insights. This means that existing methods to manage methodological flexibility can also be applied to prior elicitation. For example, multiverse analyses (Steegen et al., 2016) can be used to outline different decision paths and include them in the data analysis in a principled way. In the same way, prior sensitivity checks can be applied to test the robustness of important Bayesian modeling outcomes (e.g., the Bayes factor) to changes in the prior distributions that could be justifiable based on the elicitation results. However, existing robustness analyses such as these cannot always be used to manage the multiplicity of elicitation results. For example, not all decisions paths in prior elicitation can be taken simultaneously (e.g., experts can be selected only once) and re-computing Bayesian analyses with a multitude of prior distributions may be unfeasible for computationally intensive modeling problems. If the computational setup allows for it though, multiverse analyses and prior sensitivity checks can contribute to demonstrate the robustness of the analyses even beyond the results of the prior elicitation. For example, alternative prior distributions, such as objective prior distributions (Consonni et al., 2018), weakly informative prior distributions (Gelman et al., 2008), or empirical Bayesian priors (Casella, 1985) could be included in the analyses. This would not only present a comprehensive image of the impact of prior specification on the analysis outcomes, but also help to bridge the theoretical disagreements between different factions of Bayesian methodologists.

\section{Striving for Parsimonious Processes}

From our discussion of different decisions in prior elicitation as well as from our literature review it becomes apparent that more decisions have to be made when the prior elicitation procedure is highly complex. For example, if a researcher decides to include many experts from different backgrounds, this typically generates a series of follow-up decisions: Should the experts be allowed to interact? Should the results from different experts be combined? And if yes, how? A large number of potentially correlated parameters for which the elicitation is conducted also contributes to the complexity of the elicitation process as 
well as an indirect elicitation method where the elicited quantities are conceptually far away from the parameters of the prior distribution so that deriving the prior distribution from the elicited quantities is a complex procedure with multiple steps. Allowing for iterative adjustments of the prior distribution also leads to several follow-up decisions as has been discussed earlier in our article. Arguably, complex prior elicitation procedures are inherently challenging and should be adopted with reluctance. Therefore, as a general rule, it seems to be better to go simple than extravagant if there are no specific circumstances that require a complex elicitation procedure. Adopting a simple elicitation procedure makes the method easier to report, decreases the number of arbitrary decisions, and therefore makes the process of prior specification more robust to criticism.

\section{Preliminary Practical Recommendations for Prior Elicitation}

Throughout our paper, we have refrained from giving specific recommendations for prior elicitation from experts in practice. As we have discussed earlier, there are only few studies that compare different elicitation procedures with the goal of finding a gold standard (Grigore et al., 2016; Winkler, 1967). As a result, at the moment recommendations can only be based on theoretically evaluating the implications of methodological choices. In Table 1 we list eight preliminary recommendations that are based on such considerations and are relatively close to the issues and implications we have described earlier in this article. It goes without saying that readers should interpret these recommendations with appropriate levels of caution, even though similar recommendations can be found in the literature (e.g., O'Hagan et al., 2006; O'Hagan, 2019; Aspinall, 2010; Dias et al., 2018). We hope that the recommendations are nonetheless helpful to the reader because they provide some idea of contexts different choices in prior elicitation procedures could be commented on, for example in the context of a preregistration. 
Table 1

Preliminary recommendations for prior elicitation in practice

1. Make sure to recruit experts who have sufficient quantitative knowledge to understand the prior elicitation process.

2. Not all model parameters are equally suitable for prior elicitation. Focus on the parameters of interest and choose a parametrization that is easy to understand.

3. Be careful when combining priors from experts with widely different backgrounds because resulting prior distribution might no longer be theoretically meaningful.

4. Take measures to mitigate cognitive biases and group dynamics in the prior elicitation process.

5. Design the prior elicitation method carefully and be aware of the underlying assumptions.

6. Usually it is better to go simple than extravagant. Including many parameters and experts, eliciting quantities are conceptually far away from parameters of the prior distributions, and allowing for iterative adjustments of the elicited priors can complicate the prior elicitation process and increase the number of arbitrary decisions.

7. Prior elicitation does not result in a unique outcome. Whenever possible, conduct a sensitivity analysis for decisions made in the prior elicitation process (e.g., explore the robustness to different ways to combine elicited priors).

8. Report prior elicitation procedures transparently and completely.

\section{Conclusions}

Enriching Bayesian analysis models with knowledge of domain experts enables researchers to make realistic predictions and conduct diagnostic tests of theory even when little data are available. Therefore, informed Bayesian analyses can be advantageous in disciplines like clinical psychology, developmental psychology, or neuroscience, where data collection is often costly or time-consuming. Prior elicitation has been developed as a principled approach to specify informed prior distributions that allows researchers to incorporate knowledge of domain experts into Bayesian models. However, compared to default prior distributions, informed distributions are often considered relatively contentious (Simpson et al., 2017). Therefore, their specification needs to withstand additional scrutiny and, as a consequence, so does the prior elicitation process. In this article, we discussed several 
practical challenges that need to be addressed in the course of the planning, execution, and evaluation of the prior elicitation process. In particular, we critically examined the selection of experts and model parameters for the prior elicitation, the choice of the prior elicitation technique and the structure of the core interview process, and different ways to integrate the prior elicitation results from multiple experts. We showed how different equally justifiable elicitation decisions sometimes lead to vastly different resulting prior distributions.

To date, prior elicitation is an uncommon practice in psychological research. However, it seems plausible that the use of prior elicitation in psychological research will increase in the future. Examples of prior elicitation can already be found in diverse areas of psychological research such as social, clinical, and cognitive psychology, or psychological assessment (e.g., Al-Awadhi \& Garthwaite, 1998; Bolsinova et al., 2017; Gronau et al., in press; Tessler \& Goodman, 2019). Moreover, many statisticians and quantitative psychologists have recommended using informed priors and prior elicitation in the social sciences (e.g., Dienes, 2019; Vanpaemel, 2010). The few instances of prior elicitation in psychological applications give reason to suspect that psychology researchers are not yet fully aware of the choices that are made in the elicitation procedure (for some examples, see our online supplementary materials: https://osf.io/tbz6n/). A thorough discussion of the potentially outcome-relevant choices made in the elicitation procedure is often absent, especially when the focus of the research is on a substantial psychological topic rather than on a methodological development. We hope that our article draws researchers' attention to the importance of carefully considering these decisions and transparently reporting them in their research articles.

One reason for the high degree of methodological flexibility in prior elicitation is that little research has been done to find a gold standard. Although many elicitation techniques have been proposed, only few studies exist that compare these existing techniques with respect to their feasibility or psychometric quality (Grigore et al., 2016; Winkler, 1967). Hence, researchers are forced to make intuitive decisions between different options without the benefit of empirical evidence. Recommendations are typically based on the generalization of applied research findings, for example from research on cognitive biases 
or risk taking (e.g., O'Hagan, 2019), but are rarely based on specific research on prior elicitation for the purpose of the specification of prior distributions for Bayesian parameter estimation and hypothesis testing. A more thorough investigation of existing methods could therefore help to establish a consensus about justifiable decisions in prior elicitation and to derive evidence-based recommendations for specific application scenarios. Prior elicitation procedures bear many resemblances to other psychological methodologies that aim to assess knowledge, beliefs, or judgements, for example standard setting in educational tests (Bolsinova et al., 2017), focus groups (Kitzinger, 1995), requirement elicitation in software projects (Tiwari et al., 2012), or (semi-)structured interviews (Summerfeldt \& Antony, 2002). Further research on gold standards for prior elicitation methods could therefore be (among others) informed by evidence-based insights from these methodologies. A special focus could lie on the evidence-based factors that have been found to influence the psychometric quality, especially the validity and reliability, of these methods.

More research is also needed to compare prior elicitation from experts to other methods for the specification of informed priors in Bayesian models, such as prior specification based on previous literature (Verhagen \& Wagenmakers, 2014; Ly et al., 2019). There are some conceptual differences between these methods, for example, that prior elicitation from experts does not require comparable existing data, or that prior specification based on previous literature does not require field experts to understand the parameters of a model. However, there are arguably many situations in which different methods can be used to specify informed prior distributions, only one of which is prior elicitation from experts. There has been no research up to this point that investigates how these methods relate to each other, for example, which method is more likely to produce biased predictions, which method produces more informed prior distributions (on average), or which method is most susceptible to methodological flexibility.

It is important to note that practical methodological challenges in prior elicitation do not discredit the theoretical underpinnings of the subjective Bayesian approach. The incorporation of prior knowledge remains valuable to make the predictions of Bayesian 
models more specific and testable (e.g., Lee \& Vanpaemel, 2017). However, when outcomerelevant decisions in the prior elicitation process remain undocumented, this makes informed Bayesian approaches an easy target for criticism because no sufficient justification is provided for the prior distributions. Thus, making decisions in the prior elicitation process transparent, finding evidence-based practices that can reduce the amount of methodological flexibility, and developing new methods to deal with the remaining multiplicity of outcomes in prior elicitation can be key to a constructive advance of the debate about prior specification in Bayesian methods. 


\section{References}

Aczel, B., Hoekstra, R., Gelman, A., Wagenmakers, E. J., Klugkist, I. G., Rouder, J. N., ... van Ravenzwaaij, D. (in press). Expert opinions on how to conduct and report Bayesian inference. Nature Human Behaviour.

Aggarwal, C. C., \& Reddy, C. K. (Eds.). (2014). Data clustering: Algorithms and applications. London: CRC Press.

Al-Awadhi, S. A., \& Garthwaite, P. H. (1998). An elicitation method for multivariate normal distributions. Communications in Statistics - Theory and Methods, 27(5), 1123-1142. doi: 10 $.1080 / 03610929808832149$

Albert, I., Donnet, S., Guihenneuc-Jouyaux, C., Low-Choy, S., Mengersen, K., \& Rousseau, J. (2012). Combining expert opinions in prior elicitation. Bayesian Analysis, 7(3), 503-532. doi: $10.1214 / 12-\mathrm{ba} 717$

Andrews, M., \& Baguley, T. (2013). Prior approval: The growth of Bayesian methods in psychology. British Journal of Mathematical and Statistical Psychology, 66 (1), 1-7. doi: 10.1111/bmsp.12004

Asch, S. E. (1956). Studies of independence and conformity: I. A minority of one against a unanimous majority. Psychological Monographs: General and Applied, 70(9), 1-70. doi: 10.1037/h0093718

Aspinall, W. (2010). A route to more tractable expert advice. Nature, 463(7279), 294-295. doi: $10.1038 / 463294 a$

Baldwin, S. A., \& Fellingham, G. W. (2013). Bayesian methods for the analysis of small sample multilevel data with a complex variance structure. Psychological Methods, 18(2), 151-164. doi: $10.1037 / \mathrm{a} 0030642$

Bayarri, M. J., \& Berger, J. O. (2000). P values for composite null models. Journal of the American Statistical Association, 95(452), 1127-1142. doi: 10.2307/2669749

Bayarri, M. J., Berger, J. O., Forte, A., \& García-Donato, G. (2012). Criteria for Bayesian model choice with application to variable selection. The Annals of Statistics, 40, 1550-1577. doi: 10.1214/ 12-aos1013 
Berger, J. (2006). The case for objective Bayesian analysis. Bayesian Analysis, 1(3), 385-402. doi: 10.1214/06-BA115

Berger, R. L., \& Boos, D. D. (1994). P values maximized over a confidence set for the nuisance parameter. Journal of the American Statistical Association, 89(427), 1012-1016. doi: 10.2307/ 2290928

Boehm, U., Annis, J., Frank, M. J., Hawkins, G. E., Heathcote, A., Kellen, D., .. Wagenmakers, E.J. (2018). Estimating across-trial variability parameters of the Diffusion Decision Model: Expert advice and recommendations. Journal of Mathematical Psychology, 87, 46-75. doi: 10.1016/ j.jmp.2018.09.004

Bolger, F. (2018). The selection of experts for (probabilistic) expert knowledge elicitation. In L. C. Dias, A. Morton, \& J. Quigley (Eds.), Elicitation: The science and art of structuring judgement (p. 393-443). Springer International Publishing.

Bolsinova, M., Hoijtink, H., Vermeulen, J. A., \& Béguin, A. (2017). Using expert knowledge for test linking. Psychological Methods, 22(4), 705-724. doi: 10.1037/met0000124

Bornn, L., Doucet, A., \& Gottardo, R. (2010). An efficient computational approach for prior sensitivity analysis and cross-validation. Canadian Journal of Statistics, 1-18. doi: 10.1002/ cjs. 10045

Boyd, H. W., \& Westfall, R. (1970). Interviewer bias once more revisited. Journal of Marketing Research, 7(2), 249-253. doi: 10.2307/3150117

Casella, G. (1985). An introduction to empirical Bayes data analysis. The American Statistician, 39(2), 83-87. doi: 10.1080/00031305.1985.10479400

Chaloner, K. (1996). Elicitation of prior distributions. In D. Berry \& D. K. Stangl (Eds.), Bayesian biostatistics (p. 141-156). New York: Taylor \& Francis.

Chaloner, K., Church, T., Louis, T. A., \& Matts, J. P. (1993). Graphical elicitation of a prior distribution for a clinical trial. The Statistician, 42(4), 341. doi: 10.2307/2348469

Chow, S.-M., \& Hoijtink, H. (2017). Bayesian estimation and modeling: Editorial to the second special issue on Bayesian data analysis. Psychological Methods, 22(4), 609-615. doi: 10.1037/ met0000169 
Consonni, G., Fouskakis, D., Liseo, B., \& Ntzoufras, I. (2018). Prior distributions for objective Bayesian analysis. Bayesian Analysis, 13(2), 627-679. doi: 10.1214/18-ba1103

Crüwell, S., \& Evans, N. J. (2019). Preregistration in complex contexts: A preregistration template for the application of cognitive models. PsyArXiv Preprint. doi: 10.31234/osf.io/2hykx

Crüwell, S., Stefan, A. M., \& Evans, N. J. (2019, December). Robust standards in cognitive science. Computational Brain \& Behavior, 2(3), 255-265. doi: 10.1007/s42113-019-00049-8

Dalkey, N., \& Helmer, O. (1963). An experimental application of the DELPHI method to the use of experts. Management Science, 9(3), 458-467. doi: 10.1287/mnsc.9.3.458

DeGroot, M. H. (1974). Reaching a consensus. Journal of the American Statistical Association, 69 (345), 118. doi: $10.2307 / 2285509$

Delbecq, A. L., Van de Ven, A., \& Gustafson, D. H. (1976). Group techniques for program planning: A guide to Nominal Group and DELPHI processes. The Journal of Applied Behavioral Science, 12(4), 581-581. doi: 10.1177/002188637601200414

Depaoli, S. (2014). The impact of inaccurate "informative" priors for growth parameters in Bayesian growth mixture modeling. Structural Equation Modeling: A Multidisciplinary Journal, 21(2), 239-252. doi: 10.1080/10705511.2014.882686

Dias, L. C., Morton, A., \& Quigley, J. (Eds.). (2018). Elicitation: The science and art of structuring judgement. Springer International Publishing.

Dienes, Z. (2008). Understanding psychology as a science: An introduction to scientific and statistical inference. Houndmills: Palgrave Macmillan.

Dienes, Z. (2011). Bayesian versus orthodox statistics: Which side are you on? Perspectives on Psychological Science, 6(3), 274-290. doi: 10.1177/1745691611406920

Dienes, Z. (2019). How do I know what my theory predicts? Advances in Methods and Practices in Psychological Science, 2(4), 364-377. doi: 10.1177/2515245919876960

Dienes, Z., \& Mclatchie, N. (2017). Four reasons to prefer Bayesian analyses over significance testing. Psychonomic Bulletin \& Review, 25(1), 207-218. doi: 10.3758/s13423-017-1266-z 
Efron, B. (1986). Why isn't everyone a Bayesian? The American Statistician, 40(1), 1-5. doi: $10.2307 / 2683105$

Estes, W. K. (1956). The problem of inference from curves based on group data. Psychological Bulletin, 53(2), 134-140. doi: 10.1037/h0045156

Etz, A., Haaf, J. M., Rouder, J. N., \& Vandekerckhove, J. (2018). Bayesian inference and testing any hypothesis you can specify. Advances in Methods and Practices in Psychological Science, 1(2), 281-295. doi: $10.1177 / 2515245918773087$

Etz, A., \& Vandekerckhove, J. (2018). Introduction to Bayesian inference for psychology. Psychonomic Bulletin \& Review, 25(1), 5-34. doi: 10.3758/s13423-017-1262-3

European Food Safety Authority. (2014). Guidance on expert knowledge elicitation in food and feed safety risk assessment. EFSA Journal, 12(6). doi: 10.2903/j.efsa.2014.3734

Evans, N. J. (2019). Assessing the practical differences between model selection methods in inferences about choice response time tasks. Psychonomic Bulletin ES Review, 26(4), 1070-1098. doi: 10 $.3758 / \mathrm{s} 13423-018-01563-9$

Evans, N. J., \& Annis, J. (2019). Thermodynamic integration via differential evolution: A method for estimating marginal likelihoods. Behavior Research Methods, 51(2), 930-947. doi: 10.3758/ s13428-018-1172-y

Evans, N. J., \& Servant, M. (2020). A comparison of conflict diffusion models in the flanker task through pseudolikelihood Bayes factors. Psychological Review, 127(1), 114-135. doi: 10.1037/ rev0000165

Ferretti, V., Guney, S., Montibeller, G., \& v Winterfeldt, D. (2016). Testing best practices to reduce the overconfidence bias in multi-criteria decision analysis. In 2016 49th Hawaii International Conference on System Sciences (HICSS) (p. 1547-1555). doi: 10.1109/HICSS.2016.195

Fienberg, S. E. (2006, September). Does it make sense to be an "objective Bayesian"? (comment on articles by Berger and by Goldstein). Bayesian Analysis, 1(3), 429-432. doi: 10.1214/06-ba116c

Fink, D. (1997). A compendium of conjugate priors. https://www.johndcook.com/CompendiumOfConjugatePriors.pdf. 
Fragoso, T. M., Bertoli, W., \& Louzada, F. (2018). Bayesian Model Averaging: A systematic review and conceptual classification. International Statistical Review, 86(1), 1-28. doi: 10.1111/ insr. 12243

Garthwaite, P. H., Kadane, J. B., \& O'Hagan, A. (2005). Statistical methods for eliciting probability distributions. Journal of the American Statistical Association, 100(470), 680-701. doi: 10.1198/ 016214505000000105

Garthwaite, P. H., \& O'Hagan, A. (2000). Quantifying expert opinion in the UK water industry: An experimental study. Journal of the Royal Statistical Society: Series D (The Statistician), 49(4), 455-477. doi: 10.1111/1467-9884.00246

Gelfand, A. E., Mallick, B. K., \& Dey, D. K. (1995). Modeling expert opinion arising as a partial probabilistic specification. Journal of the American Statistical Association, 90(430), 598-604. doi: $10.1080 / 01621459.1995 .10476552$

Gelman, A., Jakulin, A., Pittau, M. G., \& Su, Y.-S. (2008). A weakly informative default prior distribution for logistic and other regression models. The Annals of Applied Statistics, 2(4), 1360-1383. doi: 10.1214/08-AOAS191

Gelman, A., Simpson, D., \& Betancourt, M. (2017). The prior can often only be understood in the context of the likelihood. Entropy, 19(10), 555. doi: 10.3390/e19100555

Genest, C., \& Zidek, J. V. (1986). Combining probability distributions: A critique and an annotated bibliography. Statistical Science, 1(1), 114-135. doi: 10.1214/ss/1177013825

Goldstein, M. (2006). Subjective Bayesian analysis: Principles and practice. Bayesian Analysis, 1(3), 403-420. doi: 10.1214/06-BA116

Gosling, J. P. (2018). SHELF: The Sheffield Elicitation Framework. In L. C. Dias, A. Morton, \& J. Quigley (Eds.), Elicitation: The science and art of structuring judgement (p. 61-94). Springer International Publishing.

Grigore, B., Peters, J., Hyde, C., \& Stein, K. (2013). Methods to elicit probability distributions from experts: A systematic review of reported practice in health technology assessment. PharmacoEconomics, 31(11), 991-1003. doi: 10.1007/s40273-013-0092-Z 
Grigore, B., Peters, J., Hyde, C., \& Stein, K. (2016). A comparison of two methods for expert elicitation in health technology assessments. BMC Medical Research Methodology, 16(1). doi: 10.1186/s12874-016-0186-3

Gronau, Q. F., Ly, A., \& Wagenmakers, E.-J. (in press). Informed Bayesian t-tests. The American Statistician. doi: 10.1080/00031305.2018.1562983

Gronau, Q. F., \& Wagenmakers, E.-J. (2019). Limitations of Bayesian leave-one-out cross-validation for model selection. Computational Brain $\&$ Behavior, 2(1), 1-11. doi: 10.1007/s42113-018-0011 $-7$

Guo, B., Park, Y., \& Liu, S. (2019). A utility-based Bayesian phase I-II design for immunotherapy trials with progression-free survival end point. Journal of the Royal Statistical Society: Series C (Applied Statistics), 68(2), 411-425. doi: 10.1111/rssc.12288

Gutenkunst, R. N., Waterfall, J. J., Casey, F. P., Brown, K. S., Myers, C. R., \& Sethna, J. P. (2007). Universally sloppy parameter sensitivities in systems biology models. PLOS Computational Biology, 3(10), 1-8. doi: 10.1371/journal.pcbi.0030189

Hanea, A. M., Burgmann, M., \& Hemming, V. (2018). IDEA for uncertainty quantification. In L. C. Dias, A. Morton, \& J. Quigley (Eds.), Elicitation: The science and art of structuring judgement (p. 95-117). Springer International Publishing.

Hartigan, J. (1964). Invariant prior distributions. The Annals of Mathematical Statistics, 35(2), 836-845. doi: 10.1214/aoms/1177703583

Head, M. L., Holman, L., Lanfear, R., Kahn, A. T., \& Jennions, M. D. (2015). The extent and consequences of p-hacking in science. PLOS Biology, 13(3), e1002106. doi: 10.1371/journal.pbio .1002106

Herrera, F., Herrera-Viedma, E., \& Verdegay, J. L. (1996). A model of consensus in group decision making under linguistic assessments. Fuzzy Sets and Systems, 78(1), 73-87. doi: 10.1016/0165 $-0114(95) 00107-7$

Hinne, M., Gronau, Q. F., van den Bergh, D., \& Wagenmakers, E.-J. (2019). A conceptual introduction to Bayesian Model Averaging. PsyArXiv Preprint. doi: 10.31234/osf.io/wgb64 
Hoeting, J. A., Madigan, D., Raftery, A. E., \& Volinsky, C. T. (1999). Bayesian model averaging: A tutorial. Statistical Science, 14(4), 382-401.

Janis, I. L. (1972). Victims of groupthink: A psychological study of foreign-policy decisions and fiascoes. Oxford, England: Houghton Mifflin.

JASP Team. (2020). JASP (Version 0.12)[Computer software]. https://jasp-stats.org/.

Jaynes, E. (1982). On the rationale of maximum-entropy methods. Proceedings of the IEEE, $70(9)$, 939-952. doi: 10.1109/proc.1982.12425

Jeffreys, H. (1939). Theory of probability (First ed.). Oxford, UK: Oxford University Press.

Jeffreys, H. (1961). Theory of probability (3rd ed.). Oxford University Press, Oxford.

Jeffreys, H. (1963). Review of "The Foundations of Statistical Inference". Technometrics, 3, 407-410.

Johnson, S. R., Tomlinson, G. A., Hawker, G. A., Granton, J. T., \& Feldman, B. M. (2010). Methods to elicit beliefs for Bayesian priors: A systematic review. Journal of Clinical Epidemiology, 63(4), 355-369. doi: 10.1016/j.jclinepi.2009.06.003

Johnson, S. R., Tomlinson, G. A., Hawker, G. A., Granton, J. T., Grosbein, H. A., \& Feldman, B. M. (2010). A valid and reliable belief elicitation method for Bayesian priors. Journal of Clinical Epidemiology, 63(4), 370-383. doi: 10.1016/j.jclinepi.2009.08.005

Kadane, J. B. (1980). Predictive and structural methods for eliciting prior distributions. In A. Zellner (Ed.), Bayesian analysis in econometrics and statistics: Essays in honor of Harold Jeffreys (Vol. 1, p. 89-93). Amsterdam: North Holland Publishing Company.

Kadane, J. B. (1986). Progress toward a more ethical method for clinical trials. The Journal of Medicine and Philosophy, 11(4), 385-404. doi: 10.1093/jmp/11.4.385

Kass, R. E., \& Raftery, A. E. (1995). Bayes factors. Journal of the American Statistical Association, 90 (430), 773-795. doi: 10.1080/01621459.1995.10476572

Kitzinger, J. (1995). Qualitative research: Introducing focus groups. BMJ, 311(7000), 299-302. doi: $10.1136 /$ bmj. 311.7000 .299 
Kumar, A., Singh, A., \& Ekavali. (2015). A review on Alzheimer's disease pathophysiology and its management: An update. Pharmacological Reports, 67(2), 195-203. doi: 10.1016/j.pharep.2014 .09 .004

Lamm, H. (1988). A review of our research on group polarization: Eleven experiments on the effects of group discussion on risk acceptance, probability estimation, and negotiation positions. Psychological Reports, 62(3), 807-813. doi: 10.2466/pr0.1988.62.3.807

Leal, J., Wordsworth, S., Legood, R., \& Blair, E. (2007). Eliciting expert opinion for economic models: An applied example. Value in Health, 10(3), 195-203. doi: 10.1111/j.1524-4733.2007 $.00169 . \mathrm{x}$

Lee, M. D., Criss, A. H., Devezer, B., Donkin, C., Etz, A., Leite, F. P., ... Vandekerckhove, J. (2019). Robust Modeling in Cognitive Science. Computational Brain \& Behavior, 2(3), 141-153. doi: 10.1007/s42113-019-00029-y

Lee, M. D., \& Vanpaemel, W. (2017). Determining informative priors for cognitive models. Psychonomic Bulletin 83 Review, 25(1), 114-127. doi: 10.3758/s13423-017-1238-3

Liang, K.-Y., \& Zeger, S. L. (1995). Inference based on estimating functions in the presence of nuisance parameters. Statistical Science, 10(2), 158-173. doi: 10.1214/ss/1177010028

Lindley, D. (1986). Comment. The American Statistician, 40(1), 6-7. doi: 10.1080/00031305.1986 .10475344

Lindley, D. (2004). That wretched prior. Significance, 1(2), 85-87. doi: 10.1111/j.1740-9713.2004 $.026 . \mathrm{x}$

Liu, C. C., \& Aitkin, M. (2008). Bayes factors: Prior sensitivity and model generalizability. Journal of Mathematical Psychology, 52(6), 362-375. doi: 10.1016/j.jmp.2008.03.002

Ly, A., Etz, A., Marsman, M., \& Wagenmakers, E.-J. (2019). Replication Bayes factors from evidence updating. Behavior Research Methods, 51(6), 2498-2508. doi: 10.3758/s13428-018-1092-x

Ly, A., Verhagen, J., \& Wagenmakers, E.-J. (2016). Harold Jeffreys's default Bayes factor hypothesis tests: Explanation, extension, and application in psychology. Journal of Mathematical Psychology, 72 (Supplement C), 19-32. doi: 10.1016/j.jmp.2015.06.004 
Montibeller, G., \& von Winterfeldt, D. (2015). Cognitive and motivational biases in decision and risk analysis. Risk Analysis, 35(7), 1230-1251. doi: 10.1111/risa.12360

Morey, R., \& Rouder, J. N. (2018). BayesFactor: Computation of Bayes factors for common designs. https://cran.r-project.org/web/packages/BayesFactor/index.html.

Morris, D. E., Oakley, J. E., \& Crowe, J. A. (2014). A web-based tool for eliciting probability distributions from experts. Environmental Modelling \& Software, 52, 1-4. doi: 10.1016/j.envsoft .2013 .10 .010

Mossman, D., Miller, W. G., Lee, E. R., Gervais, R. O., Hart, K. J., \& Wygant, D. B. (2015). A Bayesian approach to mixed group validation of performance validity tests. Psychological Assessment, 27(3), 763-776. doi: 10.1037/pas0000085

Munafò, M. R., Nosek, B. A., Bishop, D. V. M., Button, K. S., Chambers, C. D., Percie du Sert, N., ... Ioannidis, J. P. A. (2017). A manifesto for reproducible science. Nature Human Behaviour, 1(1), 0021. doi: 10.1038/s41562-016-0021

Navarro, D. J., Griffiths, T. L., Steyvers, M., \& Lee, M. D. (2006). Modeling individual differences using Dirichlet processes. Journal of Mathematical Psychology, 50(2), 101-122. doi: 10.1016/ j.jmp.2005.11.006

O'Hagan, A. (2019). Expert knowledge elicitation: Subjective but scientific. The American Statistician, 73(sup1), 69-81. doi: 10.1080/00031305.2018.1518265

O’Hagan, A., Buck, C. E., Daneshkhah, A., Eiser, R., J., Garthwaite, H., P., Jenkinson, J., D., ... Rakow, T. (2006). Uncertain judgements: Eliciting experts' probabilities. Chichester: Wiley.

O'Hagan, A., \& Oakley, J. E. (2004). Probability is perfect, but we can't elicit it perfectly. Reliability Engineering 83 System Safety, 85(1), 239-248. doi: 10.1016/j.ress.2004.03.014

O'Hagan, A., \& Oakley, J. E. (2019). SHELF - The Sheffield Elicitation Framework. Retrieved from http://tonyohagan.co.uk/shelf/

Osherson, D., Shafir, E., Krantz, D. H., \& Smith, E. E. (1997). Probability bootstrapping: Improving prediction by fitting extensional models to knowledgeable but incoherent probability judgments. Organizational Behavior and Human Decision Processes, 69(1), 1-8. doi: 10.1006/obhd.1996 .2668 
Ratcliff, R. (1979). Group reaction time distributions and an analysis of distribution statistics. Psychological Bulletin, 86(3), 446-461. doi: 10.1037/0033-2909.86.3.446

Reagan-Cirincione, P. (1994). Improving the accuracy of group judgment: A process intervention combining group facilitation, social judgment analysis, and information technology. Organizational Behavior and Human Decision Processes, 58(2), 246-270. doi: 10.1006/obhd.1994.1036

Richard, F. D., Bond, C. F., \& Stokes-Zoota, J. J. (2003). One hundred years of social psychology quantitatively described. Review of General Psychology, 7(4), 331-363. doi: 10.1037/1089-2680.7 .4 .331

Rouder, J. N., Speckman, P. L., Sun, D., Morey, R. D., \& Iverson, G. (2009). Bayesian t tests for accepting and rejecting the null hypothesis. Psychonomic Bulletin $\mathcal{E}_{3}$ Review, 16(2), 225-237. doi: $10.3758 /$ PBR.16.2.225

Rufo, M. J., Martín, J., \& Pérez, C. J. (2012). Log-linear pool to combine prior distributions: A suggestion for a calibration-based approach. Bayesian Analysis, 7(2), 411-438. doi: 10.1214/ 12-BA714

Sarma, A., \& Kay, M. (2020). Prior setting in practice: Strategies and rationales used in choosing prior distributions for Bayesian analysis. OSF Preprint. doi: 10.31219/osf.io/fcmun

Simpson, D., Rue, H., Riebler, A., Martins, T. G., \& Sørbye, S. H. (2017). Penalising model component complexity: A principled, practical approach to constructing priors. Statistical Science, 32(1), 1-28. doi: 10.1214/16-STS576

Sinharay, S., \& Stern, H. S. (2002). On the sensitivity of Bayes factors to the prior distributions. The American Statistician, 56(3), 196-201. doi: 10.1198/000313002137

Steegen, S., Tuerlinckx, F., Gelman, A., \& Vanpaemel, W. (2016). Increasing transparency through a multiverse analysis. Perspectives on Psychological Science, 11(5), 702-712. doi: $10.1177 / 1745691616658637$

Stefan, A. M., Gronau, Q. F., Schönbrodt, F. D., \& Wagenmakers, E.-J. (2019). A tutorial on Bayes Factor Design Analysis using an informed prior. Behavior Research Methods. doi: 10.3758/ s13428-018-01189-8 
Strack, F., Martin, L. L., \& Stepper, S. (1988). Inhibiting and facilitating conditions of the human smile: A nonobtrusive test of the facial feedback hypothesis. Journal of Personality and Social Psychology, 54, 768-777.

Summerfeldt, L. J., \& Antony, M. M. (2002). Structured and semistructured diagnostic interviews. In M. M. Antony \& D. H. Barlow (Eds.), Handbook of assessment and treatment planning for psychological disorders (pp. 3-37). New York: The Guilford Press.

Tendeiro, J. N., \& Kiers, H. A. L. (2019). A review of issues about null hypothesis Bayesian testing. Psychological Methods, 24(6), 774-795. doi: 10.1037/met0000221

Tessler, M. H., \& Goodman, N. D. (2019). The language of generalization. Psychological Review, 126 (3), 395-436. doi: $10.1037 /$ rev0000142

Thall, P. F., \& Cook, J. D. (2004). Dose-finding based on efficacy-toxicity trade-offs. Biometrics, 60 (3), 684-693. doi: 10.1111/j.0006-341X.2004.00218.x

Thall, P. F., Nguyen, H. Q., \& Zinner, R. G. (2017). Parametric dose standardization for optimizing two-agent combinations in a phase I-II trial with ordinal outcomes. Journal of the Royal Statistical Society: Series C (Applied Statistics), 66(1), 201-224. doi: 10.1111/rssc.12162

Tiwari, S., Rathore, S. S., \& Gupta, A. (2012). Selecting requirement elicitation techniques for software projects. In 2012 CSI Sixth International Conference on Software Engineering (CONSEG) (pp. 1-10). Indore, Madhay Pradesh, India: IEEE. doi: 10.1109/CONSEG.2012.6349486

Tversky, A., \& Kahneman, D. (1971). Belief in the law of small numbers. Psychological Bulletin, 76 (2), 105-110. doi: 10.1037/h0031322

Ursino, M., Yuan, Y., Alberti, C., Comets, E., Favrais, G., Friede, T., ... Zohar, S. (2019). A dose finding design for seizure reduction in neonates. Journal of the Royal Statistical Society: Series C (Applied Statistics), 68(2), 427-444. doi: 10.1111/rssc.12289

van Noortwijk, J., Dekker, A., Cooke, R., \& Mazzuchi, T. (1992). Expert judgment in maintenance optimization. IEEE Transactions on Reliability, 41(3), 427-432. doi: 10.1109/24.159813

van Ravenzwaaij, D., Cassey, P., \& Brown, S. D. (2016). A simple introduction to Markov Chain Monte-Carlo sampling. Psychonomic Bulletin ES Review, 25(1), 143-154. doi: 10.3758/s13423 -016-1015-8 
van Ravenzwaaij, D., \& Wagenmakers, E.-J. (2019). Advantages masquerading as 'issues' in Bayesian hypothesis testing: A commentary on Tendeiro and Kiers (2019). PsyArXiv Preprint. doi: 10 $.3124 /$ osf.io/nf7rp

Vandekerckhove, J., Rouder, J. N., \& Kruschke, J. K. (2018). Editorial: Bayesian methods for advancing psychological science. Psychonomic Bulletin \& Review, 25(1), 1-4. doi: 10.3758/ s13423-018-1443-8

Vanpaemel, W. (2010). Prior sensitivity in theory testing: An apologia for the Bayes factor. Journal of Mathematical Psychology, 54(6), 491-498. doi: 10.1016/j.jmp.2010.07.003

Verhagen, J., \& Wagenmakers, E.-J. (2014). Bayesian tests to quantify the result of a replication attempt. Journal of Experimental Psychology: General, 143(4), 1457-1475. doi: 10.1037/a0036731

Wagenmakers, E.-J., Lodewyckx, T., Kuriyal, H., \& Grasman, R. (2010). Bayesian hypothesis testing for psychologists: A tutorial on the Savage-Dickey method. Cognitive Psychology, 60(3), 158-189. doi: 10.1016/j.cogpsych.2009.12.001

Wicherts, J. M., Veldkamp, C. L. S., Augusteijn, H. E. M., Bakker, M., van Aert, R. C. M., \& van Assen, M. A. L. M. (2016). Degrees of freedom in planning, running, analyzing, and reporting psychological studies: A checklist to avoid p-hacking. Frontiers in Psychology, \%. doi: 10.3389/fpsyg.2016.01832

Wilson, K. J., \& Farrow, M. (2018). Combining judgements from correlated experts. In L. C. Dias, A. Morton, \& J. Quigley (Eds.), Elicitation: The science and art of structuring judgement (p. 211240). Springer International Publishing.

Winkler, R. L. (1967). The assessment of prior distributions in Bayesian analysis. Journal of the American Statistical Association, 62(319), 776-800. doi: 10.1080/01621459.1967.10500894

Winkler, R. L. (1980). Prior information, predictive distributions, and Bayesian model-building. In A. Zellner (Ed.), Bayesian analysis in econometrics and statistics: Essays in honor of Harold Jeffreys (Vol. 1, p. 95-109). Amsterdam: North Holland Publishing Company.

Wrinch, D., \& Jeffreys, H. (1919). On some aspects of the theory of probability. Philosophical Magazine, 38(228), 715-731. doi: 10.1080/14786441208636005 
Zellner, A., \& Min, C.-K. (1993). Bayesian analysis, model selection and prediction. In W. T. J. Grandy \& P. W. Milonni (Eds.), Physics and probability (p. 195-206). Cambridge University Press. doi: 10.1017/cbo9780511524448.019 